\title{
Non-local homogenized limits for composite media with highly anisotropic periodic fibres
}

\author{
K. D. Cherednichenko \\ St John's College, Oxford OX1 3JP, UK (cheredni@maths.ox.ac.uk) \\ V. P. Smyshlyaev \\ Department of Mathematical Sciences, University of Bath, \\ Bath BA2 7AY, UK (vps@maths.bath.ac.uk)
}

\begin{abstract}
V. V. Zhikov
Department of Mathematics, Vladimir Pedagogical University, Vladimir 600024, Russia (zhikov@vgpu.vladimir.ru)

(MS received 10 November 2004; accepted 10 May 2005)

We consider a homogenization problem for highly anisotropic conducting fibres embedded into an isotropic matrix. For a 'double porosity'-type scaling in the expression of high contrast between the conductivity along the fibres and the conductivities in the transverse directions, we prove the homogenization theorem and derive two-scale homogenized equations using a version of the method of two-scale convergence, supplemented in the case when the spectral parameter $\lambda=0$ by a newly derived variant of high-contrast Poincaré-type inequality. Further elimination of the 'rapid' component from the two-scale limit equations results in a non-local (convolution-type integro-differential) equation for the slowly varying part in the matrix, with the non-local kernel explicitly related to the Green function on the fibre. The regularity of the solution to the non-local homogenized equation is proved.
\end{abstract}

\section{Introduction}

The mathematical theory of homogenization (see, for example, $[4,5,15]$ ) establishes that in the 'classical' case, i.e. when a periodic heterogeneous medium has a moderate contrast (mathematically, is described by a uniformly elliptic partial differential equation (PDE) with rapidly oscillating coefficients), the homogenized equations preserve the local character of the original equations. The coefficients of the homogenized equations are characterized explicitly in terms of the solutions to certain 'canonical' unit-cell problems.

However, in the 'non-classical' case, i.e. when a heterogeneous medium consists of materials with highly contrasting parameters, the homogenized constitutive relation may reveal a 'non-standard' (for example, non-local) structure (see, for example, $[1,6-8,20]$, and references therein). The related studies have covered a number of settings that lead to non-local limits of various types. In spite of the large literature on the subject, there are not many contributions rigorously demonstrating explicit convolution-type spatial non-local effects for non-uniformly elliptic operators. Motivated by the study of higher-order (higher-gradient) effects in the overall 


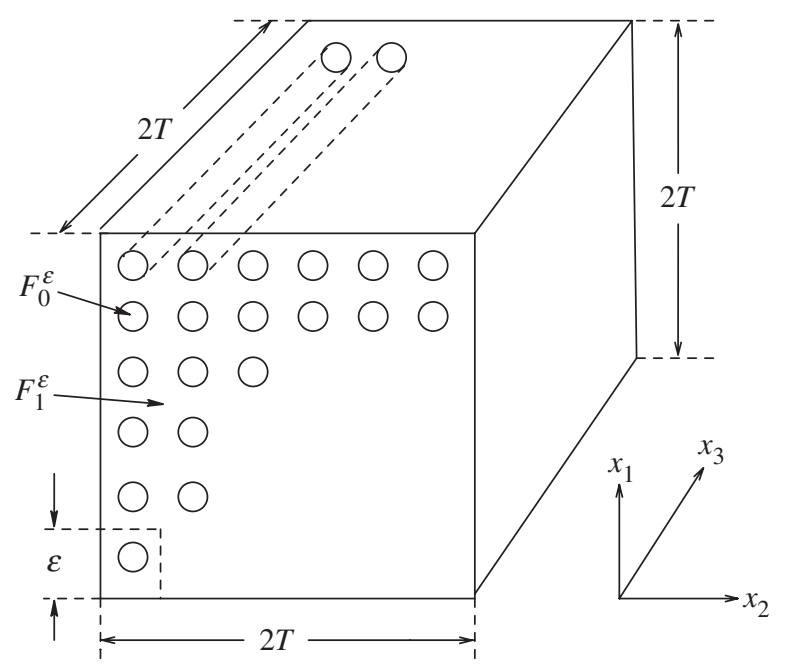

Figure 1. Geometry of the problem.

behaviour of heterogeneous media (see $[9,11,21]$ ), we consider here a special class of non-uniformly elliptic homogenization problems exhibiting this kind of limiting behaviour. Specifically, the present work concerns a homogenization problem of 'double porosity' type involving highly anisotropic fibres, with large contrast between the conductivity along the fibres and the conductivities in the transverse directions, for which the homogenized equation is an integro-differential one, displaying non-locality along the fibres. The kernel of the emerging integral operator is expressed explicitly in terms of the Green function on the fibre, and the local part is determined as in classical homogenization theory.

Earlier contributions on 'non-local' homogenization were mainly concerned with homogenization of dynamical problems, and the observed non-localities were of memory-like type, i.e. the non-local parts of the arising homogenized operators were convolution operators with respect to time $t$. In particular, Tartar [23] and Amirat et al. [3] consider homogenization of a rather general hyperbolic equation of first order and find the (weak) limit of the oscillating solutions as the small parameter $\varepsilon$ tends to zero using the Fourier and Laplace transforms. Tartar [24] rigorously proved that the limiting operator contains a convolution kernel with respect to time. Homogenization of a general class of parabolic equations with memory has been established rigorously in $[12,16]$.

A related theory was further developed by Allaire [1] and Zhikov [28], among others, who considered the problems of homogenization of an elliptic equation and a parabolic equation, respectively, with coefficients that diminish as $\varepsilon^{\gamma}, \gamma>0$, when $\varepsilon \rightarrow 0$. They showed that 'non-standard' (non-locality-type) effects arise in the critical case $\gamma=2$ (the so-called double porosity case) and derived and investigated the corresponding coupled system of homogenized equations (see also [19]).

The novelty of the present work is in investigating a non-uniformly elliptic homogenization problem whose homogenized limit exhibits an explicit non-locality due to high anisotropy in the properties of the 'components'. Namely, the non-uniform ellipticity is due to an appropriate order of contrast between the constitutive behaviour along the fibres and in the transverse directions, i.e. high anisotropy. 
More precisely, we consider a periodic composite of a fixed size $L$, whose physical properties oscillate rapidly on the scale $l \ll L$, such that $\varepsilon:=l / L$ is a small dimensionless parameter. The composite consists of a 'matrix' of a certain 'main' material, which is assumed to be connected, and (highly anisotropic) fibres included periodically into the matrix (figure 1). Section 2.1 contains a precise mathematical formulation of the problem. Preliminary analysis of the energy balance shows that, in the case when the 'anisotropy contrast' is of 'critical' order $\varepsilon^{2}$, the composite may exhibit non-standard features in the homogenized behaviour, which is demonstrated rigorously in this work. The results are obtained under rather general assumptions on the regularity properties of the fibres using the method of two-scale convergence originated by Nguetseng [18] and further developed by Allaire [1] and Zhikov [28]. (In the case when additional regularity properties are assumed, the results can be recovered by a version of an alternative method of two-scale asymptotic expansions, with additional control over the rate of convergence [10].)

We first establish the related convergence results in the case when the 'spectral parameter' $\lambda$ takes strictly positive values (see $\S 2.2$ ). This is done by combining the use of the classical Lax-Milgram lemma and the compactness property of the twoscale convergence in appropriate functional spaces (see, for example, $[1,28]$ ). As a result, we obtain a weak formulation (2.24) for the limiting homogenized boundaryvalue problem (2.26), (2.27). The latter exhibits a coupling between the slowly varying part $u^{(1)}(\boldsymbol{x})$ of the homogenized limit, corresponding to the limiting field in the matrix, and the oscillatory component $w(\boldsymbol{x}, \boldsymbol{y}), \boldsymbol{y}:=\boldsymbol{x} / \varepsilon$, which represents rapid oscillations in the fibres and vanishes in the matrix.

The case $\lambda=0$ presents additional difficulties. To overcome those, we develop a high-contrast version of the Poincaré inequality (see $\S 3$ ), specially suited to our particular setting, using some ideas of [2]. This allows us to also prove the validity of the homogenization for the case $\lambda=0$ in the original equation (2.2), from which we conclude that the homogenized equations $(2.26),(2.27)$ also hold the case of vanishing $\lambda$.

All of the above forms the basis for deriving, in $\S 4$, an integro-differential equation for the homogenized limit $u^{(1)}(\boldsymbol{x})$ by eliminating the oscillatory component $w(\boldsymbol{x}, \boldsymbol{y})$ from the coupled system. Using the methods of Fourier analysis, we demonstrate that $u^{(1)}(\boldsymbol{x})$ is infinitely smooth; hence, the homogenized equation can be considered in the classical, rather than generalized, sense. The presence of a convolution-type operator in the limiting equation can thus be viewed as a non-locality in the overall behaviour of the composite material.

\section{Statement of the problem and its homogenization}

\subsection{Formulation of the problem}

We study the problem of homogenization for two-phase electric conducting composites in which one of the constituent materials is a periodic set of highly anisotropic 'fibres' included in a 'matrix' of an isotropic conductor (figure 1). The conductivity in the fibres is assumed to be 'high' along the fibres and low in the transverse directions. (This problem serves also as a prototype, for example, for the case of an elastic composite with analogous geometry, where the fibres are considered to 
be 'stiff' in one direction and 'soft' in the orthogonal directions, while the main material is stiff in all directions.)

We next state the mathematical formulation of this problem. We introduce first a reference 'matrix-fibres' periodic medium as follows. We denote by $Q_{2}$ and $Q$ the unit cells in dimensions 2 and 3, respectively: $Q_{2}:=[0,1]^{2}$ and $Q:=Q_{2} \times$ $[0,1]$. For a $Q_{2}$-periodic set $\tilde{F}_{0} \subset \mathbb{R}^{2}$, we consider the set $F_{0}:=\tilde{F}_{0} \times \mathbb{R}$ of periodic 'fibres', and the sets $\tilde{F}_{1}:=\mathbb{R}^{2} \backslash \tilde{F}_{0}$ and $F_{1}:=\mathbb{R}^{3} \backslash F_{0}$, which correspond to the 'matrix'. Throughout the paper, unless otherwise stated, we denote the volume fractions of the phases $F_{0}$ and $F_{1}$ by $\mathfrak{f}_{0}$ and $\mathfrak{f}_{1}$, respectively; then $\mathfrak{f}_{0}=\left|\tilde{F}_{0} \cap Q_{2}\right|$ and $\mathfrak{f}_{1}=\left|\tilde{F}_{1} \cap Q_{2}\right|$. Furthermore, we fix a positive number $T$ and define $\boldsymbol{T}:=[-T, T]^{3}$. We impose periodic boundary conditions with fixed period $2 T$ to avoid dealing with boundary layers. For a small positive $\varepsilon$, such that $\varepsilon^{-1} T=: N$ is a large positive integer, we introduce contracted sets $F_{0}^{\varepsilon}:=\varepsilon F_{0}$ and $F_{1}^{\varepsilon}:=\varepsilon F_{1}$. Henceforth, $\boldsymbol{x}=\left(x_{1}, x_{2}, x_{3}\right)$ and $\boldsymbol{y}=\left(y_{1}, y_{2}, y_{3}\right)$ denote points of $\mathbb{R}^{3}$ and $Q$, respectively, and we denote by $\tilde{\boldsymbol{y}}$ and $\tilde{\boldsymbol{x}}$ the two-dimensional vectors $\left(y_{1}, y_{2}\right)$ and $\left(x_{1}, x_{2}\right)$, respectively.

We assume that the set $F_{1}$ is non-empty, open and connected. Note that under these conditions the measure $\mathrm{d} \mu_{1}:=\left.\mathrm{d} \boldsymbol{x}\right|_{F_{1}}$ (understood as the restriction of the Lebesgue measure $\mathrm{d} \boldsymbol{x}$ on $F_{1}$, continued by 0 on $\left.F_{0}\right)$ is ergodic ${ }^{1}$ and non-degenerate ${ }^{2}$, which allows us to use certain techniques originated by Zhikov [28]. (The constructions of the present paper permit further generalization by considering arbitrary ergodic Borel measures $\mu_{1}$ to allow for various 'concentration' effects [28], which we do not pursue here.)

Define a matrix function $\left(A_{i j}^{\varepsilon}(\boldsymbol{y})\right)$ by the following formula

$$
\left(A_{i j}^{\varepsilon}(\boldsymbol{y})\right)= \begin{cases}\operatorname{diag}\left(\varepsilon^{2}, \varepsilon^{2}, \alpha\right), & \text { if } \boldsymbol{y} \in F_{0}, \\ \operatorname{diag}(1,1,1)=: I, & \text { if } \boldsymbol{y} \in F_{1},\end{cases}
$$

where $\alpha>0$ is fixed. Henceforth, $\operatorname{diag}\left(a_{1}, a_{2}, a_{3}\right)$ denotes a diagonal $3 \times 3$-matrix $\left(A_{i j}\right), A_{i j}=\delta_{i j} a_{j}$ (no summation), where $\delta_{i j}$ is the Kronecker delta.

Consider an elliptic equation of 'double porosity' type:

$$
-\left(A_{i j}^{\varepsilon}\left(\frac{\boldsymbol{x}}{\varepsilon}\right) u_{, j}\right)_{, i}+\lambda u=f(\boldsymbol{x}), \quad \lambda \geqslant 0,
$$

where the comma in the subscript denotes differentiation with respect to the appropriate variable and summation is implied with respect to repeated indices. We

${ }^{1}$ A Borel measure $\mu$ is called ergodic (on the period torus generated by $Q$ ) if $u(\boldsymbol{x})=$ const. $\mu$-almost everywhere (a.e.) once there exists a sequence $\phi_{n}(\boldsymbol{x}) \in C_{\mathrm{per}}^{\infty}(Q)$ such that

$$
\int_{Q}\left|\phi_{n}(\boldsymbol{x})-u(\boldsymbol{x})\right|^{2} \mathrm{~d} \mu(\boldsymbol{x}) \rightarrow 0 \quad \text { and } \quad \int_{Q}\left|\nabla \phi_{n}(\boldsymbol{x})\right|^{2} \mathrm{~d} \mu(\boldsymbol{x}) \rightarrow 0 \quad \text { as } n \rightarrow \infty
$$

(see, for example, [28]).

${ }^{2} \mathrm{~A}$ measure $\mu$ is said to be non-degenerate (see, for example, [28]) if the zero vector is the only constant potential vector in $\left[L_{\mathrm{per}}^{2}(Q, \mathrm{~d} \mu)\right]^{3}$, which is defined as the closure of the space of $Q$-periodic infinitely differentiable functions $\phi(\boldsymbol{x})$ with respect to the norm

$$
\left(\int_{Q}|\phi(\boldsymbol{x})|^{2} \mathrm{~d} \mu(\boldsymbol{x})\right)^{1 / 2} .
$$

(For the definition of a potential vector, see [28] or footnote 14 in Appendix $\mathrm{C}$ of the present paper.) 
assume that ${ }^{3} f(\boldsymbol{x}) \in L^{2}(\boldsymbol{T}) \subset H^{-1}(\boldsymbol{T})$. In the case $\lambda=0$, we assume in addition that $\int_{\boldsymbol{T}} f(\boldsymbol{x}) \mathrm{d} \boldsymbol{x}=0$ and $\int_{\boldsymbol{T}} u(\boldsymbol{x}) \mathrm{d} \boldsymbol{x}=0$. Equation (2.2) is understood in the weak sense, that is, for a given $\varepsilon>0$, a $\boldsymbol{T}$-periodic function ${ }^{4} u^{\varepsilon}(\boldsymbol{x}) \in H_{\text {per }}^{1}(\boldsymbol{T})$ is a solution to the equation (2.2) if, for any test function $\psi(\boldsymbol{x}) \in H_{\text {per }}^{1}(\boldsymbol{T})$, the following identity holds:

$$
\begin{aligned}
\int_{\boldsymbol{T}}\left(A_{i j}^{\varepsilon}\left(\frac{\boldsymbol{x}}{\varepsilon}\right) u_{, j} \psi_{, i}+\lambda u^{\varepsilon} \psi\right) \mathrm{d} \boldsymbol{x} \\
=\int_{\boldsymbol{T} \cap F_{1}^{\varepsilon}} \nabla u^{\varepsilon} \nabla \psi \mathrm{d} \boldsymbol{x}+\varepsilon^{2} \int_{\boldsymbol{T} \cap F_{0}^{\varepsilon}}\left(u_{, 1}^{\varepsilon} \psi_{, 1}+u_{, 2}^{\varepsilon} \psi, 2\right) \mathrm{d} \boldsymbol{x} \\
\quad+\alpha \int_{\boldsymbol{T} \cap F_{0}^{\varepsilon}} u_{, 3}^{\varepsilon} \psi_{, 3} \mathrm{~d} \boldsymbol{x}+\lambda \int_{\boldsymbol{T}} u^{\varepsilon} \psi \mathrm{d} \boldsymbol{x} \\
=\int_{\boldsymbol{T}} f \psi \mathrm{d} \boldsymbol{x} .
\end{aligned}
$$

We initially consider $\lambda>0$ in (2.2), which allows us to simplify certain analyses. The case $\lambda=0$ is also considered later, and requires additional development of a certain Poincaré-type inequality for high-contrast media, which is implemented below (§3).

Before studying the behaviour of the solution of the above problem when $\varepsilon \rightarrow 0$, we give an informal idea that led us to consider equation (2.2). In [28], Zhikov studied the Cauchy problem for a parabolic equation in the two-dimensional case, as follows

$$
\begin{gathered}
\frac{\partial u^{\varepsilon}}{\partial t}(\tilde{\boldsymbol{x}}, t)-\left(a_{i j}^{\varepsilon}\left(\frac{\tilde{\boldsymbol{x}}}{\varepsilon}\right) u_{, j}^{\varepsilon}(\tilde{\boldsymbol{x}}, t)\right)_{, i}=0, \quad \tilde{\boldsymbol{x}} \in \Omega \subset \mathbb{R}^{2}, t>0, \\
u^{\varepsilon}(\tilde{\boldsymbol{x}}, 0)=f(\tilde{\boldsymbol{x}}) .
\end{gathered}
$$

Here, the entries of the $2 \times 2$-matrix $\left(a_{i j}^{\varepsilon}(\tilde{\boldsymbol{y}})\right)$ are defined by

$$
a_{i j}^{\varepsilon}(\tilde{\boldsymbol{y}})=\left(\chi_{\tilde{F}_{1}}(\tilde{\boldsymbol{y}})+\varepsilon^{2} \chi_{\tilde{F}_{0}}(\tilde{\boldsymbol{y}})\right) \delta_{i j},
$$

where $\chi_{\tilde{F}_{1}}$ and $\chi_{\tilde{F}_{0}}$ are the characteristic functions of the sets $\tilde{F}_{1}$ and $\tilde{F}_{0}$, respectively. It was shown in [28] that if $\Omega$ is a bounded domain with Lipschitz boundary, then for any $t$ the solution ${ }^{5} u^{\varepsilon} \in H_{0}^{1}(\Omega)$ of the problem (2.4), (2.5) 'two-scale converges' to the sum $u^{(1)}(\tilde{\boldsymbol{x}}, t)+v(\tilde{\boldsymbol{x}}, \tilde{\boldsymbol{y}}, t)$, where the functions $u^{(1)} \in H_{0}^{1}(\Omega)$ and $^{6}$

${ }^{3}$ For a given measure space $(\Omega, \mu)$, we define the Lebesgue class $L^{2}(\Omega, \mathrm{d} \mu)$ as the set of $\mu$ measurable functions $u(\boldsymbol{x}), \boldsymbol{x} \in \Omega$, such that the integral $\int_{\Omega}|u(\boldsymbol{x})|^{2} \mathrm{~d} \mu(\boldsymbol{x})=:\|u\|_{L^{2}}^{2} \Omega$, is well defined and finite. In particular, $L^{2}(\boldsymbol{T}):=L^{2}(\boldsymbol{T}, \mathrm{d} \boldsymbol{x})$. The class $H^{-1}(\boldsymbol{T})$ is the set of linear continuous functionals on $H_{\text {per }}^{1}(\boldsymbol{T})$; for definition of the latter, see next footnote.

${ }^{4}$ The class $H_{\text {per }}^{1}(\boldsymbol{T})$ is defined as the closure of the set of $\boldsymbol{T}$-periodic infinitely differentiable functions $\phi(\boldsymbol{x})$ with respect to the norm $\|\phi(\boldsymbol{x})\|_{L^{2}\left(\mathbb{R}^{3}\right)}+\|\nabla \phi(\boldsymbol{x})\|_{\left[L^{2}\left(\mathbb{R}^{3}\right)\right]^{3}}$.

${ }^{5}$ The class $H_{0}^{1}(\Omega)$ is defined as the closure of the set $C_{0}^{\infty}(\Omega)$ of functions $\phi(\boldsymbol{x})$ that are infinitely differentiable in $\mathbb{R}^{2}$ and that vanish outside a compact subset of the interior of $\Omega$, with respect to the norm $\|\nabla \phi(\boldsymbol{x})\|_{\left[L^{2}\left(\mathbb{R}^{3}\right)\right]^{3}}$.

${ }^{6}$ Given an arbitrary set $\Omega \in \mathbb{R}^{d}$ and a normed space $\mathcal{X}$, the class $L^{2}(\Omega, \mathcal{X})$ is defined as the class of functions $\boldsymbol{u}(\boldsymbol{x})$ with values in $\mathcal{X}$, such that the integral $\int_{\Omega}\|\boldsymbol{u}\|_{\mathcal{X}}^{2} \mathrm{~d} \boldsymbol{x}$ is well defined and finite. The definition of the space $H_{\text {per }}^{1}\left(Q_{2}\right)$ is analogous to that of the space $H_{\text {per }}^{1}(\boldsymbol{T})$ (see footnote 4$)$. 
$v \in L^{2}\left(\Omega, H_{\mathrm{per}}^{1}\left(Q_{2}\right)\right)$ satisfy the following coupled system of equations:

$$
\left.\begin{array}{c}
v_{, t}+u_{, t}^{(1)}-\Delta_{\tilde{y}} v=0, \quad \tilde{\boldsymbol{y}} \in \tilde{F}_{0} \cap Q_{2},\left.\quad v\right|_{\tilde{y} \in \tilde{F}_{1} \cap Q_{2}}=0, \\
u_{, t}^{(1)}+\langle v\rangle_{, t}-\operatorname{div}\left(A_{2 \mathrm{D}}^{\text {hom }} \nabla u^{(1)}\right)=0, \quad \tilde{\boldsymbol{x}} \in \Omega,
\end{array}\right\}
$$

together with the initial conditions

$$
u^{(1)}(\tilde{\boldsymbol{x}}, 0)=f(\tilde{\boldsymbol{x}}), \quad v(\tilde{\boldsymbol{x}}, \tilde{\boldsymbol{y}}, 0)=0 .
$$

Here, the two-dimensional homogenized matrix $A_{2 \mathrm{D}}^{\text {hom }}$ is given by

$$
A_{2 \mathrm{D}}^{\mathrm{hom}}:=\left(\begin{array}{cc}
\int_{\tilde{F}_{1} \cap Q_{2}}\left(1+\left(N_{1}\right)_{, 1}(\tilde{\boldsymbol{y}})\right) \mathrm{d} \tilde{\boldsymbol{y}} & \int_{\tilde{F}_{1} \cap Q_{2}}\left(N_{1}\right)_{, 2}(\tilde{\boldsymbol{y}}) \mathrm{d} \tilde{\boldsymbol{y}} \\
\int_{\tilde{F}_{1} \cap Q_{2}}\left(N_{2}\right)_{, 1}(\tilde{\boldsymbol{y}}) \mathrm{d} \tilde{\boldsymbol{y}} & \int_{\tilde{F}_{1} \cap Q_{2}}\left(1+\left(N_{2}\right)_{, 2}(\tilde{\boldsymbol{y}})\right) \mathrm{d} \tilde{\boldsymbol{y}}
\end{array}\right),
$$

where $N_{1}(\tilde{\boldsymbol{y}})$ and $N_{2}(\tilde{\boldsymbol{y}})$ are the solutions of the corresponding two-dimensional 'perforated' unit-cell problems in $\tilde{F}_{1} \cap Q_{2}$ with the natural (Neumann) boundary condition on $\partial \tilde{F}_{1} \cap Q_{2}$ and the periodicity conditions on the rest of $\partial\left(\tilde{F}_{1} \cap Q_{2}\right)$, and can be obtained by solving the minimization problems

$$
\min _{U} \int_{\tilde{F}_{1} \cap Q_{2}} \sum_{i=1}^{2}\left(\frac{\partial U(\boldsymbol{y})}{\partial y_{i}}+\delta_{k i}\right)^{2} \mathrm{~d} \boldsymbol{y}, \quad k=1,2,
$$

in an appropriate function space.

Note that, by expressing the function $v$ in terms of the function $u^{(1)}$ from the first of equations (2.6) and substituting it in the second, we obtain a non-local problem for $u^{(1)}$ containing a convolution operator with respect to time $t$.

Now, apply to (2.4) the Laplace transform with respect to $t$ :

$$
\hat{u}^{\varepsilon}(\tilde{\boldsymbol{x}}, \mu):=\int_{0}^{\infty} u^{\varepsilon}(\tilde{\boldsymbol{x}}, t) \exp (-\mu t) \mathrm{d} t, \quad \mu>0 .
$$

This yields

$$
-\left(a_{i j}^{\varepsilon}(\tilde{\boldsymbol{x}} / \varepsilon) \hat{u}_{, i}^{\varepsilon}(\tilde{\boldsymbol{x}})\right)_{, j}+\mu \hat{u}^{\varepsilon}(\tilde{\boldsymbol{x}})=f(\tilde{\boldsymbol{x}}), \quad \mu>0, \tilde{\boldsymbol{x}} \in \Omega \subset \mathbb{R}^{2} .
$$

If we now consider the case $\Omega=[-T, T]^{2}$ and introduce an additional spatial variable $x_{3} \in[-T, T]$, so that the function $\hat{u}^{\varepsilon}(\boldsymbol{x})$ is sought to satisfy periodic boundary conditions with respect to $\boldsymbol{x} \in \boldsymbol{T}$, then the equation (2.8) can be formally viewed as the Fourier transform with respect to $x_{3}$ of the problem (2.2) with $\alpha=1, \lambda=0$ and $f(\boldsymbol{x})=f(\tilde{\boldsymbol{x}})$, where $\mu=\xi^{2}$ and $\xi$ is the variable of the Fourier transform. Hence, formally, the parabolic equation (2.4) and the particular form of the elliptic equation (2.2) turn out to be the inverse Laplace and Fourier transforms, respectively, of the same equation, i.e. (2.8).

From this point of view, the time $t$ in (2.4) is somewhat analogous to the spatial variable $x_{3}$ in (2.2). One can expect, therefore, that the time non-locality for the system (2.6) should translate into spatial non-locality with respect to $x_{3}$ when passing to the limit $\varepsilon \rightarrow 0$ in the equation (2.2). This is indeed the case, as has 
been rigorously established in the present work. Note, however, that the methods we develop are more general, i.e. they are valid even when the Fourier transform is not applicable (for $\alpha \neq 1$ in (2.2)).

Moreover, the techniques developed in the present paper remain capable of accounting for a somewhat more general case of anisotropy of the fibres than the one represented by (2.1). Namely, in $(2.2), A_{i j}^{\varepsilon}(\boldsymbol{x} / \varepsilon)$ can be replaced by $A_{i j}^{\varepsilon}(\boldsymbol{x}, \boldsymbol{x} / \varepsilon)$, with a 'two-scale' matrix $\left(A_{i j}^{\varepsilon}(\boldsymbol{x}, \boldsymbol{y})\right)$ of a more general form than (2.1). For example, one may assume that, while being periodic with respect to $\boldsymbol{y}$ for every $\boldsymbol{x}$, it satisfies the 'usual' uniform ellipticity conditions in the matrix phase $F_{1}$ :

$$
\nu|\boldsymbol{\eta}|^{2} \leqslant A_{i j}^{\varepsilon}(\boldsymbol{x}, \boldsymbol{y}) \eta_{i} \eta_{j} \leqslant \nu^{-1}|\boldsymbol{\eta}|^{2}
$$

for some positive $\varepsilon$-independent constant $\nu$, any $\boldsymbol{\eta} \in \mathbb{R}^{3}, \boldsymbol{x} \in \boldsymbol{T}$ and $\boldsymbol{y} \in Q$, and a 'relaxed' anisotropic ellipticity condition in the fibres $F_{0}$ :

$$
\nu\left[\varepsilon^{2}\left(\eta_{1}^{2}+\eta_{2}^{2}\right)+\eta_{3}^{2}\right] \leqslant A_{i j}^{\varepsilon}(\boldsymbol{x}, \boldsymbol{y}) \eta_{i} \eta_{j} \leqslant \nu^{-1}\left[\varepsilon^{2}\left(\eta_{1}^{2}+\eta_{2}^{2}\right)+\eta_{3}^{2}\right] .
$$

The condition (2.10) means that $A^{\varepsilon}(\boldsymbol{x}, \boldsymbol{y})=\left(A_{i j}^{\varepsilon}(\boldsymbol{x}, \boldsymbol{y})\right)$ can be represented as follows:

$$
A^{\varepsilon}(\boldsymbol{x}, \boldsymbol{y})=\left(\begin{array}{ccc}
\varepsilon^{2} B_{11}^{\varepsilon} & \varepsilon^{2} B_{12}^{\varepsilon} & \varepsilon B_{13}^{\varepsilon} \\
\varepsilon^{2} B_{21}^{\varepsilon} & \varepsilon^{2} B_{22}^{\varepsilon} & \varepsilon B_{23}^{\varepsilon} \\
\varepsilon B_{31}^{\varepsilon} & \varepsilon B_{32}^{\varepsilon} & B_{33}^{\varepsilon}
\end{array}\right),
$$

where $B^{\varepsilon}(\boldsymbol{x}, \boldsymbol{y})=\left(B_{i j}^{\varepsilon}(\boldsymbol{x}, \boldsymbol{y})\right)$ satisfies the 'classical' ellipticity conditions (2.9) with $A_{i j}^{\varepsilon}$ replaced by $B_{i j}^{\varepsilon}$. In $(2.11)$ the terms $B_{12}^{\varepsilon}=B_{21}^{\varepsilon}$ represent possible rotation of the 'principal axes' of the conductivity tensor in the transverse plane, and the terms $B_{13}^{\varepsilon}=B_{31}^{\varepsilon}$ and $B_{23}^{\varepsilon}=B_{32}^{\varepsilon}$ correspond to a possible slight deviation of the 'main' axis of anisotropy from the $x_{3}$-direction (more precisely, the angle between this principal axis and the direction of the fibres is $O(\varepsilon)$ as $\varepsilon \rightarrow 0$ ) and the values of the moduli that are $O(1)$ in the direction of the axis and $O\left(\varepsilon^{2}\right)$ in the transverse directions. The dependence of $A_{i j}^{\varepsilon}$ on $\boldsymbol{x}$ reflects the possibility of non-trivial dependence on the slow as well the fast variables ( $\boldsymbol{x}$ and $\boldsymbol{x} / \varepsilon$, respectively), both in the matrix $F_{1}^{\varepsilon}$ and on the fibres $F_{0}^{\varepsilon}$, while keeping the order of anisotropy of the fibres as before. We claim that all the methodology we employ in this paper remains applicable to this generalized case too, including the emergence of non-locality in the homogenized limit as $\varepsilon \rightarrow 0$. The above generalization will not, in our opinion, lead to additional difficulties in the mathematical treatment: in particular, all the a priori estimates, for example, (2.12), below, remain valid. Extending the related calculations to include these extra features will present no challenge to a motivated reader and, since dealing with the matrix (2.1) facilitates the exposition of the main ideas and results, we refrain from elaborating further in this direction.

\subsection{Passing to the limit in equation (2.2) when $\varepsilon \rightarrow 0$}

In this section we assume that $\lambda>0$ in (2.2). Using the classical Lax-Milgram lemma (see, for example, $[15,27]$ ) it is not difficult to see that, for every $\varepsilon>0$, there exists a unique solution $u^{\varepsilon}$ to the equation $(2.2)$ in the class $H_{\text {per }}^{1}(\boldsymbol{T})$.

To find the limiting, or homogenized, behaviour of the solution $u^{\varepsilon}$ when $\varepsilon \rightarrow 0$ we will implement the method of two-scale convergence, introduced by Nguetseng [18] 
and further developed by Allaire [1] and Zhikov [28]. Basic facts about the two-scale convergence are reviewed in Appendix A for the reader's convenience. With the aim of employing a compactness argument, we first prove that the expressions $u^{\varepsilon}$ and $\varepsilon \nabla u^{\varepsilon}$ are bounded in $L^{2}(\boldsymbol{T})$, uniformly with respect to $\varepsilon \in(0,1]$, i.e.

$$
\int_{\boldsymbol{T}}\left|u^{\varepsilon}\right|^{2} \mathrm{~d} \boldsymbol{x} \leqslant C, \quad \varepsilon^{2} \int_{\boldsymbol{T}}\left|\nabla u^{\varepsilon}\right|^{2} \mathrm{~d} \boldsymbol{x} \leqslant C,
$$

where $C$ is a constant independent of $\varepsilon$ but possibly dependent on $\lambda$. Taking $\psi=u^{\varepsilon}$ in $(2.3)$, we get

$$
\begin{aligned}
\int_{\boldsymbol{T} \cap F_{1}^{\varepsilon}}\left|\nabla u^{\varepsilon}\right|^{2} \mathrm{~d} \boldsymbol{x}+\varepsilon^{2} \int_{\boldsymbol{T} \cap F_{0}^{\varepsilon}}\left(\left(u_{, 1}^{\varepsilon}\right)^{2}+\left(u_{, 2}^{\varepsilon}\right)^{2}\right) \mathrm{d} \boldsymbol{x} & \\
& +\alpha \int_{\boldsymbol{T} \cap F_{0}^{\varepsilon}}\left(u_{, 3}^{\varepsilon}\right)^{2} \mathrm{~d} \boldsymbol{x}+\lambda \int_{\boldsymbol{T}}\left(u^{\varepsilon}\right)^{2} \mathrm{~d} \boldsymbol{x}=\int_{\boldsymbol{T}} f u^{\varepsilon} \mathrm{d} \boldsymbol{x} .
\end{aligned}
$$

Hence,

$$
\begin{aligned}
\int_{\boldsymbol{T} \cap F_{1}^{\varepsilon}}\left|\nabla u^{\varepsilon}\right|^{2} \mathrm{~d} \boldsymbol{x} & +\varepsilon^{2} \int_{\boldsymbol{T} \cap F_{0}^{\varepsilon}}\left(\left(u_{, 1}^{\varepsilon}\right)^{2}+\left(u_{, 2}^{\varepsilon}\right)^{2}\right) \mathrm{d} \boldsymbol{x}+\alpha \int_{\boldsymbol{T} \cap F_{0}^{\varepsilon}}\left(u_{, 3}^{\varepsilon}\right)^{2} \mathrm{~d} \boldsymbol{x}+\lambda \int_{\boldsymbol{T}}\left(u^{\varepsilon}\right)^{2} \mathrm{~d} \boldsymbol{x} \\
& \leqslant\left(\int_{\boldsymbol{T}} f^{2} \mathrm{~d} \boldsymbol{x}\right)^{1 / 2}\left(\int_{\boldsymbol{T}}\left(u^{\varepsilon}\right)^{2} \mathrm{~d} \boldsymbol{x}\right)^{1 / 2} \leqslant \frac{1}{2 \lambda} \int_{\boldsymbol{T}} f^{2} \mathrm{~d} \boldsymbol{x}+\frac{\lambda}{2} \int_{\boldsymbol{T}}\left(u^{\varepsilon}\right)^{2} \mathrm{~d} \boldsymbol{x},
\end{aligned}
$$

and, therefore,

$$
\begin{aligned}
\int_{\boldsymbol{T} \cap F_{1}^{\varepsilon}}\left|\nabla u^{\varepsilon}\right|^{2} \mathrm{~d} \boldsymbol{x}+\varepsilon^{2} \int_{\boldsymbol{T} \cap F_{0}^{\varepsilon}}\left(\left(u_{, 1}^{\varepsilon}\right)^{2}+\left(u_{, 2}^{\varepsilon}\right)^{2}\right) \mathrm{d} \boldsymbol{x} \\
+\alpha \int_{\boldsymbol{T} \cap F_{0}^{\varepsilon}}\left(u_{, 3}^{\varepsilon}\right)^{2} \mathrm{~d} \boldsymbol{x}+\frac{\lambda}{2} \int_{\boldsymbol{T}}\left(u^{\varepsilon}\right)^{2} \mathrm{~d} \boldsymbol{x} \leqslant \frac{1}{2 \lambda} \int_{\boldsymbol{T}} f^{2} \mathrm{~d} \boldsymbol{x}
\end{aligned}
$$

which implies (2.12).

Using the compactness property of two-scale convergence (see, for example, $[1,18]$; Appendix A), we deduce that, up to a subsequence,

$$
u^{\varepsilon}(\boldsymbol{x}) \stackrel{2}{\rightarrow} u(\boldsymbol{x}, \boldsymbol{y}) \in L^{2}\left(\boldsymbol{T}, H_{\mathrm{per}}^{1}(Q)\right)
$$

and

$$
\varepsilon \nabla u^{\varepsilon}(\boldsymbol{x}) \stackrel{2}{\rightarrow} \nabla_{y} u(\boldsymbol{x}, \boldsymbol{y}),
$$

where the notation ' $\stackrel{2}{ }$ ', stands for the (weak) two-scale convergence. We note in passing that the latter convergence statement implies that, on denoting the characteristic function of the set $F_{1}$ by $\chi_{1}(\boldsymbol{y})$, we have

$$
\varepsilon \chi_{1}\left(\varepsilon^{-1} \boldsymbol{x}\right) \nabla u^{\varepsilon}(\boldsymbol{x}) \stackrel{2}{\rightarrow} \chi_{1}(\boldsymbol{y}) \nabla_{y} u(\boldsymbol{x}, \boldsymbol{y}),
$$

using the property of multiplication by a function for two-scale convergence (see, for example, [28] or Appendix A). 
On the other hand, it is clear from (2.13) that the expression $\chi_{1}\left(\varepsilon^{-1} \boldsymbol{x}\right) \nabla u^{\varepsilon}(\boldsymbol{x})$ is bounded in $L^{2}(\boldsymbol{T})$ and therefore $\varepsilon \chi_{1}\left(\varepsilon^{-1} \boldsymbol{x}\right) \nabla u^{\varepsilon}(\boldsymbol{x}) \rightarrow 0$ in $\left[L^{2}(\boldsymbol{T})\right]^{3}$ strongly. Hence,

$$
\varepsilon \chi_{1}\left(\varepsilon^{-1} \boldsymbol{x}\right) \nabla u^{\varepsilon}(\boldsymbol{x}) \stackrel{2}{\rightarrow} 0 .
$$

Comparing (2.16) and (2.17), we conclude that

$$
\chi_{1}(\boldsymbol{y}) \nabla_{y} u(\boldsymbol{x}, \boldsymbol{y})=\left.\nabla_{y} u(\boldsymbol{x}, \boldsymbol{y})\right|_{y \in F_{1} \cap Q}=0 .
$$

Therefore, $\left.u(\boldsymbol{x}, \boldsymbol{y})\right|_{y \in F_{1} \cap Q}=u^{(1)}(\boldsymbol{x})$ for some function $u^{(1)}(\boldsymbol{x}) \in L^{2}(\boldsymbol{T})$, by the ergodicity of $F_{1}$. Moreover, we prove in Appendix B that $u^{(1)}(\boldsymbol{x}) \in H_{\text {per }}^{1}(\boldsymbol{T})$.

In the same fashion, from $(2.13), \varepsilon u_{3}^{\varepsilon}(\boldsymbol{x}) \rightarrow 0$ in $L^{2}(\boldsymbol{T})$ and hence $u_{y_{3}}(\boldsymbol{x}, \boldsymbol{y})=0$. Thus, we conclude that $u \in V$, where the function space $V$ is defined by the formula ${ }^{7}$

$$
V=\left\{u(\boldsymbol{x}, \tilde{\boldsymbol{y}}) \in L^{2}\left(\boldsymbol{T}, H_{\mathrm{per}}^{1}\left(Q_{2}\right)\right):\left.u\right|_{\tilde{y} \in \tilde{F}_{1} \cap Q_{2}}=u^{(1)}(\boldsymbol{x}) \in H_{\mathrm{per}}^{1}(\boldsymbol{T})\right\} .
$$

To perform the passage to the two-scale limit, let us next consider, in (2.3), the test functions $\psi$ of a particular type: $\psi(\boldsymbol{x})=\psi^{\varepsilon}(\boldsymbol{x})=\Phi\left(\boldsymbol{x}, \varepsilon^{-1} \tilde{\boldsymbol{x}}\right)$, where $\Phi(\boldsymbol{x}, \tilde{\boldsymbol{y}})$ is of the form

$$
\Phi(\boldsymbol{x}, \tilde{\boldsymbol{y}})=\Phi_{1}(\boldsymbol{x})+\beta(\boldsymbol{x}) h(\tilde{\boldsymbol{y}}) .
$$

Here $\Phi_{1}(\boldsymbol{x}), \beta(\boldsymbol{x}) \in C_{\text {per }}^{\infty}(\boldsymbol{T})$ and $h \in X:=\left\{h(\tilde{\boldsymbol{y}}) \in C_{\text {per }}^{\infty}\left(Q_{2}\right),\left.h\right|_{\tilde{F}_{1} \cap Q_{2}}=0\right\}$. Obviously,

$$
\left.\nabla \psi^{\varepsilon}(\boldsymbol{x})\right|_{\boldsymbol{T} \cap F_{1}^{\varepsilon}}=\left.\nabla \Phi_{1}(\boldsymbol{x})\right|_{\boldsymbol{T} \cap F_{1}^{\varepsilon}}
$$

and

$$
\begin{aligned}
\varepsilon \nabla \psi^{\varepsilon}(\boldsymbol{x}) & =\varepsilon \nabla \Phi_{1}(\boldsymbol{x})+\varepsilon \nabla \beta(\boldsymbol{x}) h\left(\varepsilon^{-1} \tilde{\boldsymbol{x}}\right)+\left.\beta(\boldsymbol{x}) \nabla_{y} h(\tilde{\boldsymbol{y}})\right|_{y=\varepsilon^{-1} x} \\
& =\left.\beta(\boldsymbol{x}) \nabla_{y} h(\tilde{\boldsymbol{y}})\right|_{y=\varepsilon^{-1} x}+o(1)
\end{aligned}
$$

as $\varepsilon \rightarrow 0$, where $o(1)$ is understood in the sense of the $L^{2}(\boldsymbol{T})$ norm. Substituting $\psi=\psi^{\varepsilon}$ into the identity $(2.3)$, we get

$$
\begin{aligned}
\int_{\boldsymbol{T} \cap F_{1}^{\varepsilon}} & \nabla u^{\varepsilon}(\boldsymbol{x}) \nabla \Phi_{1}(\boldsymbol{x}) \mathrm{d} \boldsymbol{x} \\
& +\varepsilon \int_{\boldsymbol{T} \cap F_{0}^{\varepsilon}}\left(\left.u_{, 1}^{\varepsilon}(\boldsymbol{x}) \beta(\boldsymbol{x}) h_{, y_{1}}(\tilde{\boldsymbol{y}})\right|_{\tilde{y}=\varepsilon^{-1} x}+\left.u_{, 2}^{\varepsilon}(\boldsymbol{x}) \beta(\boldsymbol{x}) h_{, y_{2}}(\tilde{\boldsymbol{y}})\right|_{\tilde{y}=\varepsilon^{-1} x}\right) \mathrm{d} \boldsymbol{x} \\
& +\varepsilon \int_{\boldsymbol{T} \cap F_{0}^{\varepsilon}}\left(u_{, 1}^{\varepsilon}(\boldsymbol{x}) o(1)+u_{, 2}^{\varepsilon}(\boldsymbol{x}) o(1)\right) \mathrm{d} \boldsymbol{x}+\alpha \int_{\boldsymbol{T} \cap F_{0}^{\varepsilon}} u_{, 3}^{\varepsilon}(\boldsymbol{x}) \Phi_{, x_{3}}\left(\boldsymbol{x}, \varepsilon^{-1} \tilde{\boldsymbol{x}}\right) \mathrm{d} \boldsymbol{x} \\
& +\lambda \int_{\boldsymbol{T}} u^{\varepsilon}(\boldsymbol{x}) \Phi\left(\boldsymbol{x}, \varepsilon^{-1} \tilde{\boldsymbol{x}}\right) \mathrm{d} \boldsymbol{x} \\
= & \int_{\boldsymbol{T}} f(\boldsymbol{x}) \Phi\left(\boldsymbol{x}, \varepsilon^{-1} \tilde{\boldsymbol{x}}\right) \mathrm{d} \boldsymbol{x} .
\end{aligned}
$$

${ }^{7}$ In the case of a more general ergodic Borel measure $\mu_{1}$ (see the discussion preceding (2.1)), the space

$$
\begin{aligned}
& V=\left\{u(\boldsymbol{x}, \tilde{\boldsymbol{y}}) \in L^{2}\left(\boldsymbol{T}, H_{\mathrm{per}}^{1}\left(Q_{2}, \mathrm{~d} \mu_{1}\right)\right):\right. \\
& \left.\left.u\right|_{\tilde{y} \in \tilde{F}_{1} \cap Q_{2}}=u^{(1)}(\boldsymbol{x}) \in H_{\mathrm{per}}^{1}(\boldsymbol{T}),\left.\nabla_{\tilde{y}} u\right|_{\tilde{y} \in \tilde{F}_{1} \cap Q_{2}}=\tilde{\mathbf{0}} \text { a.e. } \boldsymbol{x} \in \boldsymbol{T}\right\}
\end{aligned}
$$

should be used instead, where $H_{\text {per }}^{1}\left(Q_{2}, \mathrm{~d} \mu_{1}\right)$ is an appropriately defined Sobolev space with respect to the measure $\mu_{1}$ (see, for example, [28]). 
We want next to pass to the limit when $\varepsilon \rightarrow 0$ in the identity (2.19). Firstly, note that, as shown in Appendix C,

$$
\lim _{\varepsilon \rightarrow 0} \int_{\boldsymbol{T} \cap F_{1}^{\varepsilon}} \nabla u^{\varepsilon}(\boldsymbol{x}) \nabla \Phi_{1}(\boldsymbol{x}) \mathrm{d} \boldsymbol{x}=\int_{\boldsymbol{T}} A_{1}^{\mathrm{hom}} \nabla u^{(1)}(\boldsymbol{x}) \nabla \Phi_{1}(\boldsymbol{x}) \mathrm{d} \boldsymbol{x},
$$

where $A_{1}^{\text {hom }}$ is the homogenized matrix for 'hollow' fibres (cf. (2.7)):

$$
A_{1}^{\mathrm{hom}}:=\left(\begin{array}{ccc}
\int_{\tilde{F}_{1} \cap Q_{2}}\left(1+\left(N_{1}\right)_{, 1}(\tilde{\boldsymbol{y}})\right) \mathrm{d} \tilde{\boldsymbol{y}} & \int_{\tilde{F}_{1} \cap Q_{2}}\left(N_{1}\right)_{, 2}(\tilde{\boldsymbol{y}}) \mathrm{d} \tilde{\boldsymbol{y}} & 0 \\
\int_{\tilde{F}_{1} \cap Q_{2}}\left(N_{2}\right)_{, 1}(\tilde{\boldsymbol{y}}) \mathrm{d} \tilde{\boldsymbol{y}} & \int_{\tilde{F}_{1} \cap Q_{2}}\left(1+\left(N_{2}\right)_{, 2}(\tilde{\boldsymbol{y}})\right) \mathrm{d} \tilde{\boldsymbol{y}} & 0 \\
0 & 0 & \mathfrak{f}_{1}
\end{array}\right) .
$$

Secondly, using (2.15) we obtain

$$
\begin{aligned}
\varepsilon \int_{\boldsymbol{T} \cap F_{0}^{\varepsilon}}\left(\left.u_{, 1}^{\varepsilon}(\boldsymbol{x}) \beta(\boldsymbol{x}) h_{, 1}(\tilde{\boldsymbol{y}})\right|_{\tilde{y}=\varepsilon^{-1} \tilde{x}}+\left.u_{, 2}^{\varepsilon}(\boldsymbol{x}) \beta(\boldsymbol{x}) h_{, 2}(\tilde{\boldsymbol{y}})\right|_{\tilde{y}=\varepsilon^{-1}} \tilde{x}\right) \mathrm{d} \boldsymbol{x} \\
=\left.\varepsilon \int_{\boldsymbol{T}}\left(u_{, 1}^{\varepsilon}(\boldsymbol{x}) \beta(\boldsymbol{x}) h_{, 1}(\tilde{\boldsymbol{y}}) \chi_{0}(\tilde{\boldsymbol{y}})+u_{, 2}^{\varepsilon}(\boldsymbol{x}) \beta(\boldsymbol{x}) h_{, 2}(\tilde{\boldsymbol{y}}) \chi_{0}(\tilde{\boldsymbol{y}})\right)\right|_{\tilde{y}=\varepsilon^{-1} \tilde{x}} \mathrm{~d} \boldsymbol{x} \\
\quad \stackrel{\varepsilon \rightarrow 0}{\longrightarrow} \int_{\boldsymbol{T}} \int_{Q_{2}}\left(u_{, y_{1}}(\boldsymbol{x}, \tilde{\boldsymbol{y}}) \beta(\boldsymbol{x}) h_{, 1}(\tilde{\boldsymbol{y}}) \chi_{0}(\tilde{\boldsymbol{y}})+u_{, y_{2}}(\boldsymbol{x}, \tilde{\boldsymbol{y}}) \beta(\boldsymbol{x}) h_{, 2}(\tilde{\boldsymbol{y}}) \chi_{0}(\tilde{\boldsymbol{y}})\right) \mathrm{d} \tilde{\boldsymbol{y}} \mathrm{d} \boldsymbol{x} \\
=\int_{\boldsymbol{T}} \int_{\tilde{F}_{0} \cap Q_{2}}\left(u_{, y_{1}}(\boldsymbol{x}, \tilde{\boldsymbol{y}}) \beta(\boldsymbol{x}) h_{, 1}(\tilde{\boldsymbol{y}})+u_{, y_{2}}(\boldsymbol{x}, \tilde{\boldsymbol{y}}) \beta(\boldsymbol{x}) h_{, 2}(\tilde{\boldsymbol{y}})\right) \mathrm{d} \tilde{\boldsymbol{y}} \mathrm{d} \boldsymbol{x} \\
=\int_{\boldsymbol{T}} \int_{\tilde{F}_{0} \cap Q_{2}}\left(u_{, y_{1}}(\boldsymbol{x}, \tilde{\boldsymbol{y}}) \Phi_{, y_{1}}(\boldsymbol{x}, \tilde{\boldsymbol{y}})+u_{, y_{2}}(\boldsymbol{x}, \tilde{\boldsymbol{y}}) \Phi_{, y_{2}}(\boldsymbol{x}, \tilde{\boldsymbol{y}})\right) \mathrm{d} \tilde{\boldsymbol{y}} \mathrm{d} \boldsymbol{x} .
\end{aligned}
$$

In the formula above, $\chi_{0}$ denotes the characteristic function of the set $\tilde{F}_{0} \cap Q_{2}$.

Furthermore, due to the fact that $\varepsilon \nabla u^{\varepsilon}$ is bounded in $L^{2}(\boldsymbol{T})$, the following convergence holds:

$$
\varepsilon \int_{\boldsymbol{T} \cap F_{0}^{\varepsilon}}\left(u_{, 1}^{\varepsilon}(\boldsymbol{x}) o(1)+u_{, 2}^{\varepsilon}(\boldsymbol{x}) o(1)\right) \mathrm{d} \boldsymbol{x} \stackrel{\varepsilon \rightarrow 0}{\longrightarrow} 0 .
$$

Finally, we find the limit of the term

$$
\alpha \int_{\boldsymbol{T} \cap F_{0}^{\varepsilon}} u_{, 3}^{\varepsilon}(\boldsymbol{x}) \Phi_{, x_{3}}\left(\boldsymbol{x}, \varepsilon^{-1} \tilde{\boldsymbol{x}}\right) \mathrm{d} \boldsymbol{x}
$$

as $\varepsilon \rightarrow 0$. Note that the inequality (2.13) implies the estimate

$$
\int_{\boldsymbol{T}}\left(u_{, 3}^{\varepsilon}\right)^{2} \mathrm{~d} \boldsymbol{x} \leqslant C
$$

and therefore there exists such a function $q(\boldsymbol{x}, \boldsymbol{y}) \in L^{2}(\boldsymbol{T} \times Q)$ that, up to a subsequence,

$$
u_{, 3}^{\varepsilon} \stackrel{2}{\rightarrow} q(\boldsymbol{x}, \boldsymbol{y}) .
$$

In order to obtain the limit of the expression (2.22), we establish the following lemma. 
LEMma 2.1. Let $u(\boldsymbol{x}, \tilde{\boldsymbol{y}}) \in V$ and $q(\boldsymbol{x}, \boldsymbol{y})$ be the limiting functions from (2.14) and (2.23). Then the Sobolev derivative $u_{x_{3}}(\boldsymbol{x}, \tilde{\boldsymbol{y}}) \in L^{2}\left(\boldsymbol{T} \times Q_{2}\right)$ exists and is given by the equality $u_{, x_{3}}(\boldsymbol{x}, \tilde{\boldsymbol{y}})=\langle q(\boldsymbol{x}, \boldsymbol{y})\rangle_{y_{3}}$, where

$$
\langle q(\boldsymbol{x}, \boldsymbol{y})\rangle_{y_{3}}:=\int_{0}^{1} q(\boldsymbol{x}, \boldsymbol{y}) \mathrm{d} y_{3} .
$$

Proof. Assume that $\Psi=\Psi(\boldsymbol{x}, \tilde{\boldsymbol{y}}) \in C_{\mathrm{per}}^{\infty}\left(\boldsymbol{T}, C_{\mathrm{per}}^{\infty}\left(Q_{2}\right)\right)$. By means of integration by parts we may write

$$
\int_{\boldsymbol{T}} u_{, 3}^{\varepsilon}(\boldsymbol{x}) \Psi\left(\boldsymbol{x}, \varepsilon^{-1} \tilde{\boldsymbol{x}}\right) \mathrm{d} \boldsymbol{x}=-\int_{\boldsymbol{T}} u^{\varepsilon}(\boldsymbol{x}) \Psi_{, x_{3}}\left(\boldsymbol{x}, \varepsilon^{-1} \tilde{\boldsymbol{x}}\right) \mathrm{d} \boldsymbol{x} .
$$

Using convergences (2.14) and (2.23), we pass to the limit as $\varepsilon \rightarrow 0$ in the above formula, and obtain the following identity:

$$
\int_{\boldsymbol{T}} \int_{Q} q(\boldsymbol{x}, \boldsymbol{y}) \Psi(\boldsymbol{x}, \tilde{\boldsymbol{y}}) \mathrm{d} \boldsymbol{y} \mathrm{d} \boldsymbol{x}=-\int_{\boldsymbol{T}} \int_{Q_{2}} u(\boldsymbol{x}, \tilde{\boldsymbol{y}}) \Psi_{, x_{3}}(\boldsymbol{x}, \tilde{\boldsymbol{y}}) \mathrm{d} \tilde{\boldsymbol{y}} \mathrm{d} \boldsymbol{x} .
$$

Clearly, it can be rewritten as

$$
\int_{\boldsymbol{T}} \int_{Q_{2}}\langle q(\boldsymbol{x}, \boldsymbol{y})\rangle_{y_{3}} \Psi(\boldsymbol{x}, \tilde{\boldsymbol{y}}) \mathrm{d} \tilde{\boldsymbol{y}} \mathrm{d} \boldsymbol{x}=-\int_{\boldsymbol{T}} \int_{Q_{2}} u(\boldsymbol{x}, \tilde{\boldsymbol{y}}) \Psi_{, x_{3}}(\boldsymbol{x}, \tilde{\boldsymbol{y}}) \mathrm{d} \tilde{\boldsymbol{y}} \mathrm{d} \boldsymbol{x} .
$$

By the definition of Sobolev derivative, this implies the statement of the lemma.

Now, employing the above lemma, we get

$$
\begin{aligned}
& \int_{\boldsymbol{T} \cap F_{0}^{\varepsilon}} u_{, 3}^{\varepsilon}(\boldsymbol{x}) \Phi\left(\boldsymbol{x}, \varepsilon^{-1} \tilde{\boldsymbol{x}}\right) \mathrm{d} \boldsymbol{x} \stackrel{\varepsilon \rightarrow \infty}{\longrightarrow} \int_{\boldsymbol{T}} \int_{Q} q(\boldsymbol{x}, \boldsymbol{y}) \Phi_{, x_{3}}(\boldsymbol{x}, \tilde{\boldsymbol{y}}) \mathrm{d} \boldsymbol{y} \mathrm{d} \boldsymbol{x} \\
&=\int_{\boldsymbol{T}} \int_{Q_{2}}\langle q(\boldsymbol{x}, \boldsymbol{y})\rangle_{y_{3}} \Phi_{, x_{3}}(\boldsymbol{x}, \tilde{\boldsymbol{y}}) \mathrm{d} \tilde{\boldsymbol{y}} \mathrm{d} \boldsymbol{x} \\
&=\int_{\boldsymbol{T}} \int_{Q_{2}} u_{, x_{3}}(\boldsymbol{x}, \tilde{\boldsymbol{y}}) \Phi_{, x_{3}}(\boldsymbol{x}, \tilde{\boldsymbol{y}}) \mathrm{d} \tilde{\boldsymbol{y}} \mathrm{d} \boldsymbol{x} .
\end{aligned}
$$

Using the convergence results obtained, we pass to the limit in (2.19) as $\varepsilon \rightarrow 0$, which yields

$$
\begin{aligned}
\int_{\boldsymbol{T}} A_{1}^{\mathrm{hom}} & \nabla u^{(1)}(\boldsymbol{x}) \nabla \Phi_{1}(\boldsymbol{x}) \mathrm{d} \boldsymbol{x} \\
& +\int_{\boldsymbol{T}} \int_{\tilde{F}_{0} \cap Q_{2}}\left(u_{, y_{1}}(\boldsymbol{x}, \tilde{\boldsymbol{y}}) \Phi_{y_{1}}(\boldsymbol{x}, \tilde{\boldsymbol{y}})+u_{y_{2}}(\boldsymbol{x}, \tilde{\boldsymbol{y}}) \Phi_{y_{2}}(\boldsymbol{x}, \tilde{\boldsymbol{y}})\right) \mathrm{d} \tilde{\boldsymbol{y}} \mathrm{d} \boldsymbol{x} \\
& +\alpha \int_{\boldsymbol{T}} \int_{Q_{2}} u_{x_{3}}(\boldsymbol{x}, \tilde{\boldsymbol{y}}) \Phi_{, x_{3}}(\boldsymbol{x}, \tilde{\boldsymbol{y}}) \mathrm{d} \tilde{\boldsymbol{y}} \mathrm{d} \boldsymbol{x}+\lambda \int_{\boldsymbol{T}} \int_{Q_{2}} u(\boldsymbol{x}, \tilde{\boldsymbol{y}}) \Phi(\boldsymbol{x}, \tilde{\boldsymbol{y}}) \mathrm{d} \tilde{\boldsymbol{y}} \mathrm{d} \boldsymbol{x} \\
= & \int_{\boldsymbol{T}} \int_{Q} f(\boldsymbol{x}) \Phi(\boldsymbol{x}, \tilde{\boldsymbol{y}}) \mathrm{d} \boldsymbol{y} \mathrm{d} \boldsymbol{x},
\end{aligned}
$$

for any function $\Phi$ of the form (2.18).

The identity (2.24) is naturally equivalent to a system of two partial differential equations. To see this, we first take $\Phi=\Phi_{1}(\boldsymbol{x}) \in C_{\mathrm{per}}^{\infty}(\boldsymbol{T})$ in (2.24) and obtain the 
equality

$$
\begin{aligned}
\int_{\boldsymbol{T}} A_{1}^{\mathrm{hom}} \nabla u^{(1)}(\boldsymbol{x}) \nabla \Phi_{1}(\boldsymbol{x}) \mathrm{d} \boldsymbol{x}+\alpha \int_{\boldsymbol{T}} \int_{\tilde{F}_{0} \cap Q_{2}} u_{, x_{3}}(\boldsymbol{x}, \tilde{\boldsymbol{y}})\left(\Phi_{1}\right)_{, 3}(\boldsymbol{x}) \mathrm{d} \tilde{\boldsymbol{y}} \mathrm{d} \boldsymbol{x} \\
+\lambda \int_{\boldsymbol{T}} \int_{Q_{2}} u(\boldsymbol{x}, \tilde{\boldsymbol{y}}) \Phi_{1}(\boldsymbol{x}) \mathrm{d} \tilde{\boldsymbol{y}} \mathrm{d} \boldsymbol{x}=\int_{\boldsymbol{T}} \int_{Q} f(\boldsymbol{x}) \Phi_{1}(\boldsymbol{x}) \mathrm{d} \boldsymbol{y} \mathrm{d} \boldsymbol{x},
\end{aligned}
$$

which can be rewritten in the following way, denoting $w(\boldsymbol{x}, \tilde{\boldsymbol{y}}):=u(\boldsymbol{x}, \tilde{\boldsymbol{y}})-u^{(1)}(\boldsymbol{x})$ (hence, $w(\boldsymbol{x}, \tilde{\boldsymbol{y}})=0$ for $\left.\tilde{\boldsymbol{y}} \in \tilde{F}_{1}\right)$ :

$$
\begin{gathered}
\int_{\boldsymbol{T}} A_{1}^{\mathrm{hom}} \nabla u^{(1)}(\boldsymbol{x}) \nabla \Phi_{1}(\boldsymbol{x}) \mathrm{d} \boldsymbol{x}+\alpha \mathfrak{f}_{0} \int_{\boldsymbol{T}} u_{, 3}^{(1)}(\boldsymbol{x})\left(\Phi_{1}\right)_{, 3}(\boldsymbol{x}) \mathrm{d} \boldsymbol{x} \\
+\alpha \int_{\boldsymbol{T}}\langle w\rangle_{, 3}(\boldsymbol{x})\left(\Phi_{1}\right)_{, 3}(\boldsymbol{x}) \mathrm{d} \boldsymbol{x}+\lambda \int_{\boldsymbol{T}}\left(u^{(1)}(\boldsymbol{x})+\langle w\rangle(\boldsymbol{x})\right) \Phi_{1}(\boldsymbol{x}) \mathrm{d} \boldsymbol{x} \\
=\int_{\boldsymbol{T}} f(\boldsymbol{x}) \Phi_{1}(\boldsymbol{x}) \mathrm{d} \boldsymbol{x},
\end{gathered}
$$

where $\langle w\rangle(\boldsymbol{x}):=\int_{Q} w(\boldsymbol{x}, \tilde{\boldsymbol{y}}) \mathrm{d} \boldsymbol{y}$. Note that the last identity holds for any $\Phi_{1}(\boldsymbol{x}) \in$ $C_{\text {per }}^{\infty}(\boldsymbol{T})$. Therefore, it is equivalent to

$$
-\operatorname{div}\left(A^{\mathrm{hom}} \nabla u^{(1)}\right)-\alpha \frac{\partial^{2}\langle w\rangle}{\partial x_{3}^{2}}+\lambda\left(u^{(1)}+\langle w\rangle\right)=f,
$$

where the matrix $A^{\text {hom }}$ is defined by the following formula (cf. (2.21) and (2.7))

$$
A^{\mathrm{hom}}:=\left(\begin{array}{ccc}
\int_{\tilde{F}_{1} \cap Q_{2}}\left(1+\left(N_{1}\right)_{, 1}(\tilde{\boldsymbol{y}})\right) \mathrm{d} \tilde{\boldsymbol{y}} & \int_{\tilde{F}_{1} \cap Q_{2}}\left(N_{1}\right)_{, 2}(\tilde{\boldsymbol{y}}) \mathrm{d} \tilde{\boldsymbol{y}} & 0 \\
\int_{\tilde{F}_{1} \cap Q_{2}}\left(N_{2}\right)_{, 1}(\tilde{\boldsymbol{y}}) \mathrm{d} \tilde{\boldsymbol{y}} & \int_{\tilde{F}_{1} \cap Q_{2}}\left(1+\left(N_{2}\right)_{, 2}(\tilde{\boldsymbol{y}})\right) \mathrm{d} \tilde{\boldsymbol{y}} & 0 \\
0 & 0 & \mathfrak{f}_{1}+\alpha \mathfrak{f}_{0}
\end{array}\right) .
$$

Now, taking $\Phi(\boldsymbol{x}, \tilde{\boldsymbol{y}})=\beta(\boldsymbol{x}) h(\tilde{\boldsymbol{y}})$, where $\beta(\boldsymbol{x}) \in C_{\mathrm{per}}^{\infty}(\boldsymbol{T})$ and $h(\tilde{\boldsymbol{y}}) \in X$, and substituting it into the identity $(2.24)$, we get

$$
\begin{gathered}
\int_{\boldsymbol{T}} \int_{\tilde{F}_{0} \cap Q_{2}}\left(w_{, y_{1}}(\boldsymbol{x}, \tilde{\boldsymbol{y}}) h_{, 1}(\tilde{\boldsymbol{y}})+w_{, y_{2}}(\boldsymbol{x}, \tilde{\boldsymbol{y}}) h_{, 2}(\tilde{\boldsymbol{y}})\right) \beta(\boldsymbol{x}) \mathrm{d} \tilde{\boldsymbol{y}} \mathrm{d} \boldsymbol{x} \\
+\alpha \int_{\boldsymbol{T}} \int_{\tilde{F}_{0} \cap Q_{2}} u_{, x_{3}}(\boldsymbol{x}, \tilde{\boldsymbol{y}}) h(\tilde{\boldsymbol{y}}) \beta_{, 3}(\boldsymbol{x}) \mathrm{d} \tilde{\boldsymbol{y}} \mathrm{d} \boldsymbol{x} \\
\quad+\lambda \int_{\boldsymbol{T}} \int_{\tilde{F}_{0} \cap Q_{2}} u(\boldsymbol{x}, \tilde{\boldsymbol{y}}) h(\tilde{\boldsymbol{y}}) \beta(\boldsymbol{x}) \mathrm{d} \tilde{\boldsymbol{y}} \mathrm{d} \boldsymbol{x} \\
=\int_{\boldsymbol{T}} \int_{Q} f(\boldsymbol{x}) h(\tilde{\boldsymbol{y}}) \beta(\boldsymbol{x}) \mathrm{d} \boldsymbol{y} \mathrm{d} \boldsymbol{x} .
\end{gathered}
$$

The last equality holds for any $\beta(\boldsymbol{x}) \in C_{\text {per }}^{\infty}(\boldsymbol{T})$ and $h(\tilde{\boldsymbol{y}}) \in C_{\text {per }}^{\infty}\left(Q_{2}\right),\left.h\right|_{\tilde{y} \in \tilde{F}_{1} \cap Q_{2}}=0$. Hence, it is equivalent to the partial differential equation

$$
-\frac{\partial^{2} w}{\partial y_{1}^{2}}-\frac{\partial^{2} w}{\partial y_{2}^{2}}-\alpha \frac{\partial^{2} w}{\partial x_{3}^{2}}-\alpha \frac{\partial^{2} u^{(1)}}{\partial x_{3}^{2}}+\lambda\left(u^{(1)}+w\right)=f, \quad \tilde{\boldsymbol{y}} \in \tilde{F}_{0} \cap Q_{2} .
$$


Summarizing, we conclude that the following theorem holds.

TheOREM 2.2. Let $\lambda>0$. Then, for the solutions $u^{\varepsilon}(\boldsymbol{x})$ of the problems (2.2),

$$
u^{\varepsilon}(\boldsymbol{x}) \stackrel{2}{\rightarrow} u^{(1)}(\boldsymbol{x})+w(\boldsymbol{x}, \tilde{\boldsymbol{y}}) \quad \text { as } \varepsilon \rightarrow 0,
$$

where the $\boldsymbol{T}$-periodic (with respect to $\boldsymbol{x}$ ) functions $u^{(1)}$ and $w$ satisfy (in the weak sense) the following system of elliptic equations:

$$
\left.\begin{array}{cc}
-\operatorname{div}\left(A^{\mathrm{hom}} \nabla u^{(1)}\right)-\alpha \frac{\partial^{2}\langle w\rangle}{\partial x_{3}^{2}}+\lambda\left(u^{(1)}+\langle w\rangle\right)=f, & \boldsymbol{x} \in \boldsymbol{T}, \\
\frac{\partial^{2} w}{\partial y_{1}^{2}}-\frac{\partial^{2} w}{\partial y_{2}^{2}}-\alpha \frac{\partial^{2} w}{\partial x_{3}^{2}}-\alpha \frac{\partial^{2} u^{(1)}}{\partial x_{3}^{2}}+\lambda\left(u^{(1)}+w\right)=f, & \tilde{\boldsymbol{y}} \in \tilde{F}_{0} \cap Q_{2},
\end{array}\right\}
$$

together with the boundary condition ${ }^{8}$

$$
\left.w(\boldsymbol{x}, \tilde{\boldsymbol{y}})\right|_{\tilde{y} \in \tilde{F}_{1} \cap Q_{2}}=0 .
$$

Note that, due to the uniqueness of the weak solution of (2.26), (2.27) (equivalently, of the function $u(\boldsymbol{x}, \boldsymbol{y})$ satisfying $(2.24)$ ), the whole 'sequence' $u^{\varepsilon}(\boldsymbol{x})$ two-scale converges to the function $u^{(1)}(\boldsymbol{x})+w(\boldsymbol{x}, \tilde{\boldsymbol{y}})$.

\section{The case $\lambda=0$}

Before studying further the homogenized system (2.26), we argue that the parameter $\lambda$ in the original equation (2.2) can be set to zero ${ }^{9}$ without altering the validity of the stated results. In particular, we argue that in this case the homogenized equation (2.26), with $\lambda=0$, still holds. ${ }^{10}$ However, establishing this requires some additional analysis. The result follows from the next theorem, which establishes a version of Poincaré's inequality, adapted to the present high-contrast case under study.

TheOREM 3.1. Let $\boldsymbol{T}=[-T, T]^{d}, T>0$ and $Q=[0,1]^{d}$. Let $F_{0}$ be a $Q$-periodic set with Lipschitz boundary such that $F_{1}=\mathbb{R}^{d} \backslash F_{0}$ is non-empty, open and connected; $F_{0}^{\varepsilon}=\varepsilon F_{0}$ and $F_{1}^{\varepsilon}=\varepsilon F_{1}$, where $\varepsilon>0$ is such that $\varepsilon^{-1} T=: N$ is a positive integer.

There then exists an $\varepsilon$-independent positive constant $C$ such that, for any function $u \in H^{1}(\boldsymbol{T})$ with zero mean over $\boldsymbol{T}$, the following Poincaré-type inequality holds:

$$
\|u\|_{L^{2}(\boldsymbol{T})} \leqslant C\left(\|\nabla u\|_{L^{2}\left(\boldsymbol{T} \cap F_{1}^{\varepsilon}\right)}+\varepsilon\|\nabla u\|_{L^{2}\left(\boldsymbol{T} \cap F_{0}^{\varepsilon}\right)}\right) .
$$

Proof. Here we use some ideas presented by Allaire and Murat [2].

${ }^{8}$ In addition, the function $w(\boldsymbol{x}, \tilde{\boldsymbol{y}})$ is required to be $Q_{2}$-periodic in $\tilde{\boldsymbol{y}}$.

${ }^{9}$ Note that in this case the function $f(\boldsymbol{x})$ is assumed to have zero mean over $\boldsymbol{T}$ and the solution $u(\boldsymbol{x})$ is sought in the class $H_{0, \mathrm{per}}^{1}(\boldsymbol{T})$ of the functions from $H_{0}^{1}(\boldsymbol{T})$ that have zero mean over $\boldsymbol{T}$; for the definition of $H_{0}^{1}(\boldsymbol{T})$ see footnote 5 .

${ }^{10}$ Of course, in the case $\lambda=0$, in addition to the condition (2.27), one more constraint should be imposed on the unknown functions $u^{(1)}$ and $w$, namely $\int_{\boldsymbol{T}}\left(u^{(1)}(\boldsymbol{x})+\langle w\rangle(\boldsymbol{x})\right) \mathrm{d} \boldsymbol{x}=0$. We get this condition by observing that $\int_{\boldsymbol{T}} u^{\varepsilon}(\boldsymbol{x}) \mathrm{d} \boldsymbol{x} \rightarrow \int_{\boldsymbol{T}} \int_{Q}\left(u_{1}(\boldsymbol{x})+w(\boldsymbol{x}, \tilde{\boldsymbol{y}})\right) \mathrm{d} \boldsymbol{y} \mathrm{d} \boldsymbol{x}$ due to the two-scale convergence (2.25). 
Throughout the proof we use the following notation:

$$
\langle g\rangle_{D}:=|D|^{-1} \int_{D} g(\boldsymbol{x}) \mathrm{d} \boldsymbol{x},
$$

where $D$ is a bounded measurable set in $\mathbb{R}^{d}$ and the function $g$ belongs to the space $L^{1}(D)$. Let us list some useful basic properties of the operation of averaging $\langle\cdot\rangle_{D}$. First, this operation is linear: for any $g_{1}, g_{2} \in L^{1}(D)$,

$$
\left\langle g_{1}+g_{2}\right\rangle_{D}=\left\langle g_{1}\right\rangle_{D}+\left\langle g_{2}\right\rangle_{D} .
$$

Next, if $D=D_{0} \cup D_{1}$ is a partition of the set $D$ into two disjoint subsets $D_{0}$ and $D_{1}$, and $\mathfrak{f}_{0}=|D|^{-1}\left|D_{0}\right|$ and $\mathfrak{f}_{1}=|D|^{-1}\left|D_{1}\right|$ are their volume fractions, then

$$
\langle g\rangle_{D}=\mathfrak{f}_{0}\langle g\rangle_{D_{0}}+\mathfrak{f}_{1}\langle g\rangle_{D_{1}} .
$$

Finally, if $g \in L^{2}(D)$, then the following well-known inequality holds:

$$
\left\langle g^{2}\right\rangle_{D} \geqslant\langle g\rangle_{D}^{2}
$$

Now, partition the cube $\boldsymbol{T}$ into disjoint cubes $Q_{\varepsilon}^{i}, i=1, \ldots, K_{\varepsilon}$, of size $\varepsilon$, where $K_{\varepsilon}:=(2 N)^{d}$, and consider two piecewise constant functions on $\boldsymbol{T}$ :

$$
\bar{u}(\boldsymbol{x})=\langle u\rangle_{Q_{\varepsilon}^{i}}, \boldsymbol{x} \in Q_{\varepsilon}^{i},
$$

and

$$
\tilde{u}(\boldsymbol{x})=\langle u\rangle_{Q_{\varepsilon}^{i} \cap F_{1}^{\varepsilon}}, \boldsymbol{x} \in Q_{\varepsilon}^{i} .
$$

Using the triangle inequality in $L^{2}(\boldsymbol{T})$, we get

$$
\|u\|_{L^{2}(\boldsymbol{T})} \leqslant\|u-\bar{u}\|_{L^{2}(\boldsymbol{T})}+\|\bar{u}-\tilde{u}\|_{L^{2}(\boldsymbol{T})}+\|\tilde{u}\|_{L^{2}(\boldsymbol{T})} .
$$

Let us estimate separately each of the terms in (3.5). For the first term we obtain

$$
\begin{aligned}
\|u-\bar{u}\|_{L^{2}(\boldsymbol{T})}^{2} & =\sum_{i=1}^{K_{\varepsilon}} \int_{Q_{\varepsilon}^{i}}\left|u(\boldsymbol{x})-\langle u\rangle_{Q_{\varepsilon}^{i}}\right|^{2} \mathrm{~d} \boldsymbol{x} \\
& =\varepsilon^{d} \sum_{i=1}^{K_{\varepsilon}} \int_{\varepsilon^{-1} Q_{\varepsilon}^{i}}\left|u\left(\varepsilon \boldsymbol{x}^{\prime}\right)-\left\langle u\left(\varepsilon \boldsymbol{x}^{\prime}\right)\right\rangle_{x^{\prime} \in \varepsilon^{-1} Q_{\varepsilon}^{i}}\right|^{2} \mathrm{~d} \boldsymbol{x}^{\prime} \\
& \leqslant \varepsilon^{d} C_{P}(Q) \sum_{i=1}^{K_{\varepsilon}} \int_{\varepsilon^{-1} Q_{\varepsilon}^{i}}\left|\nabla_{x^{\prime}} u\left(\varepsilon \boldsymbol{x}^{\prime}\right)\right|^{2} \mathrm{~d} \boldsymbol{x}^{\prime} \\
& =\varepsilon^{2} C_{P}(Q) \sum_{i=1}^{K_{\varepsilon}} \int_{Q_{\varepsilon}^{i}}|\nabla u(\boldsymbol{x})|^{2} \mathrm{~d} \boldsymbol{x} \\
& =\varepsilon^{2} C_{P}(Q)\|\nabla u\|_{L^{2}(\boldsymbol{T})}^{2} .
\end{aligned}
$$

Here we split the original integral into a sum of $K_{\varepsilon}$ constituent integrals, then rescale the variable of integration; after that we use the standard Poincaré inequality (3.10) for each cell $\varepsilon^{-1} Q_{\varepsilon}^{i}$, and finally rescale the variable of integration back. Thus,

$$
\|u-\bar{u}\|_{L^{2}(\boldsymbol{T})} \leqslant C_{1} \varepsilon\|\nabla u\|_{L^{2}(\boldsymbol{T})},
$$

where $C_{1}=\sqrt{C_{P}(Q)}$. 
In what follows, we require the following lemma.

LEMMA 3.2. Let $\Omega$ be a bounded, open and connected set in $\mathbb{R}^{d}$ with a Lipschitz boundary. Then, for any partition $\Omega=\Omega_{0} \cup \Omega_{1}$ of the domain $\Omega$ into two disjoint subsets $\Omega_{0}$ and $\Omega_{1}$ with non-zero volumes, there exists a positive constant $C=C\left(\Omega_{0}, \Omega_{1}\right)$ such that, for any function $v \in H^{1}(\Omega)$, the following inequality holds:

$$
\left|\langle v\rangle_{\Omega_{0}}-\langle v\rangle_{\Omega_{1}}\right| \leqslant C\|\nabla v\|_{L^{2}(\Omega)} .
$$

Proof. Note first that, if we denote the volume fractions $|\Omega|^{-1}\left|\Omega_{0}\right|$ and $|\Omega|^{-1}\left|\Omega_{1}\right|$ of the constituent subsets $\Omega_{0}$ and $\Omega_{1}$ by $\mathfrak{f}_{0}$ and $\mathfrak{f}_{1}$, respectively, then

$$
\begin{aligned}
\left\langle\left|v-\langle v\rangle_{\Omega}\right|^{2}\right\rangle_{\Omega} & =\mathfrak{f}_{0}\left\langle\left|v-\langle v\rangle_{\Omega}\right|^{2}\right\rangle_{\Omega_{0}}+\mathfrak{f}_{1}\left\langle\left|v-\langle v\rangle_{\Omega}\right|^{2}\right\rangle_{\Omega_{1}} \\
& \geqslant \mathfrak{f}_{0}\left\langle\left|v-\langle v\rangle_{\Omega}\right|\right\rangle_{\Omega_{0}}^{2}+\mathfrak{f}_{1}\left\langle\left|v-\langle v\rangle_{\Omega}\right|\right\rangle_{\Omega_{1}}^{2} \\
& \geqslant \mathfrak{f}_{0}\left\langle v-\langle v\rangle_{\Omega}\right\rangle_{\Omega_{0}}^{2}+\mathfrak{f}_{1}\left\langle v-\langle v\rangle_{\Omega}\right\rangle_{\Omega_{1}}^{2} \\
& =\mathfrak{f}_{0}\left(\langle v\rangle_{\Omega_{0}}-\langle v\rangle_{\Omega}\right)^{2}+\mathfrak{f}_{1}\left(\langle v\rangle_{\Omega_{1}}-\langle v\rangle_{\Omega}\right)^{2} \\
& =\mathfrak{f}_{0} \mathfrak{f}_{1}^{2}\left(\langle v\rangle_{\Omega_{0}}-\langle v\rangle_{\Omega_{1}}\right)^{2}+\mathfrak{f}_{1} \mathfrak{f}_{0}^{2}\left(\langle v\rangle_{\Omega_{1}}-\langle v\rangle_{\Omega_{0}}\right)^{2} \\
& =\mathfrak{f}_{0} \mathfrak{f}_{1}\left(\langle v\rangle_{\Omega_{0}}-\langle v\rangle_{\Omega_{1}}\right)^{2} .
\end{aligned}
$$

In (3.8), we use (3.3) with $D_{0}=\Omega_{0}$ and $D_{1}=\Omega_{1}$, the property (3.4) with $D=\Omega_{0}$ and then with $D=\Omega_{1}$, and (3.2) with $D=\Omega_{0}$ and $D=\Omega_{1}$. After that we use (3.3) once again, followed by the trivial fact that $\mathfrak{f}_{0}+\mathfrak{f}_{1}=1$. Thus, it is proved that

$$
\left(\langle v\rangle_{\Omega_{0}}-\langle v\rangle_{\Omega_{1}}\right)^{2} \leqslant\left(\mathfrak{f}_{0} \mathfrak{f}_{1}\right)^{-1}\left\langle\left|v-\langle v\rangle_{\Omega}\right|^{2}\right\rangle_{\Omega} .
$$

Now, using the formula (3.9) and the classical Poincaré inequality

$$
\left\langle\left|v-\langle v\rangle_{\Omega}\right|^{2}\right\rangle_{\Omega} \leqslant C_{P}(\Omega)\|\nabla v\|_{L^{2}(\Omega)}^{2},
$$

we obtain

$$
\left(\langle v\rangle_{\Omega_{0}}-\langle v\rangle_{\Omega_{1}}\right)^{2} \leqslant\left(\mathfrak{f}_{0} \mathfrak{f}_{1}\right)^{-1} C_{P}(\Omega)\|\nabla v\|_{L^{2}(\Omega)}^{2},
$$

which implies (3.7) with $C=\sqrt{\left(\mathfrak{f}_{0} \mathfrak{f}_{1}\right)^{-1} C_{P}(\Omega)}$.

The second term in the right-hand side of (3.5) is now estimated as follows:

$$
\begin{aligned}
\|\bar{u}-\tilde{u}\|_{L^{2}(\boldsymbol{T})}^{2} & =\sum_{i=1}^{K_{\varepsilon}} \int_{Q_{\varepsilon}^{i}}\left(\langle u\rangle_{Q_{\varepsilon}^{i}}-\langle u\rangle_{Q_{\varepsilon}^{i} \cap F_{1}^{\varepsilon}}\right)^{2} \mathrm{~d} \boldsymbol{x}=\varepsilon^{d} \sum_{i=1}^{K_{\varepsilon}}\left(\langle u\rangle_{Q_{\varepsilon}^{i}}-\langle u\rangle_{Q_{\varepsilon}^{i} \cap F_{1}^{\varepsilon}}\right)^{2} \\
& =\varepsilon^{d} \mathfrak{f}_{0}^{2} \sum_{i=1}^{K_{\varepsilon}}\left(\langle u\rangle_{Q_{\varepsilon}^{i} \cap F_{0}^{\varepsilon}}-\langle u\rangle_{Q_{\varepsilon}^{i} \cap F_{1}^{\varepsilon}}\right)^{2} \\
& =\varepsilon^{d} \mathfrak{f}_{0}^{2} \sum_{i=1}^{K_{\varepsilon}}\left(\left\langle u\left(\varepsilon \boldsymbol{x}^{\prime}\right)\right\rangle_{x^{\prime} \in \varepsilon^{-1} Q_{\varepsilon}^{i} \cap F_{0}}-\left\langle u\left(\varepsilon \boldsymbol{x}^{\prime}\right)\right\rangle_{x^{\prime} \in \varepsilon^{-1} Q_{\varepsilon}^{i} \cap F_{1}}\right)^{2} \\
& \leqslant \varepsilon^{d} \mathfrak{f}_{0} \mathfrak{f}_{1}^{-1} C_{P}(Q) \sum_{i=1}^{K_{\varepsilon}} \int_{\varepsilon^{-1} Q_{\varepsilon}^{i}}\left|\nabla_{x^{\prime}} u\left(\varepsilon \boldsymbol{x}^{\prime}\right)\right|^{2} \mathrm{~d} \boldsymbol{x}^{\prime}
\end{aligned}
$$




$$
\begin{aligned}
& =\mathfrak{f}_{0} \mathfrak{f}_{1}^{-1} C_{P}(Q) \varepsilon^{2} \sum_{i=1}^{K_{\varepsilon}} \int_{Q_{\varepsilon}^{i}}|\nabla u(\boldsymbol{x})|^{2} \mathrm{~d} \boldsymbol{x} \\
& =\mathfrak{f}_{0} \mathfrak{f}_{1}^{-1} C_{P}(Q) \varepsilon^{2} \int_{\boldsymbol{T}}|\nabla u(\boldsymbol{x})|^{2} \mathrm{~d} \boldsymbol{x}=\mathfrak{f}_{0} \mathfrak{f}_{1}^{-1} C_{P}(Q) \varepsilon^{2}\|\nabla u\|_{L^{2}(\boldsymbol{T})}^{2},
\end{aligned}
$$

where $\mathfrak{f}_{0}=\left|F_{0} \cap Q\right|$ and $\mathfrak{f}_{1}=\left|F_{1} \cap Q\right|$. In formula (3.12) we split the original expression into a sum of $K_{\varepsilon}$ integrals over cells of size $\varepsilon$ and use the fact that every integration in (3.12) is performed on a constant function. Then we make use of (3.3), where $D_{0}=Q_{\varepsilon}^{i} \cap F_{0}^{\varepsilon}$ and $D_{1}=Q_{\varepsilon}^{i} \cap F_{1}^{\varepsilon}$, and rescale the variable of integration to obtain the expression in (3.13), which we estimate using inequality (3.11) with $\Omega=Q$. Finally, in (3.14) we rescale the variable of integration back and get (3.15) by the additivity of the integral. ${ }^{11}$ Hence, it is proved that

$$
\|\bar{u}-\tilde{u}\|_{L^{2}(\boldsymbol{T})} \leqslant C_{2} \varepsilon\|\nabla u\|_{L^{2}(\boldsymbol{T})},
$$

where $C_{2}=\sqrt{\mathfrak{f}_{0} \mathfrak{f}_{1}^{-1} C_{P}(Q)}$. Note that

$$
\|\bar{u}-\tilde{u}\|_{L^{2}(\boldsymbol{T})}^{2}=\sum_{i=1}^{K_{\varepsilon}} \int_{Q_{\varepsilon}^{i}}\left|\langle u\rangle_{Q_{\varepsilon}^{i}}-\langle u\rangle_{Q_{\varepsilon}^{i} \cap F_{1}^{\varepsilon}}\right|^{2} \mathrm{~d} \boldsymbol{x}=\varepsilon^{d} \sum_{i=1}^{K_{\varepsilon}}\left|\langle u\rangle_{Q_{\varepsilon}^{i}}-\langle u\rangle_{Q_{\varepsilon}^{i} \cap F_{1}^{\varepsilon}}\right|^{2},
$$

and therefore it follows that

$$
\sum_{i=1}^{K_{\varepsilon}}\left|\langle u\rangle_{Q_{\varepsilon}^{i}}-\langle u\rangle_{Q_{\varepsilon}^{i} \cap F_{1}^{\varepsilon}}\right|^{2} \leqslant C_{2}^{2} \varepsilon^{2-d}\|\nabla u\|_{L^{2}(\boldsymbol{T})}^{2} .
$$

Finally, we estimate the third term in the right-hand side of (3.5). To this end, note that, due to the fact that

$$
\sum_{i=1}^{K_{\varepsilon}}\langle u\rangle_{Q_{\varepsilon}^{i}}=(2 N)^{d}\langle u\rangle_{\boldsymbol{T}}=0
$$

the following formulae hold:

$$
\begin{aligned}
\left|\sum_{i=1}^{K_{\varepsilon}}\langle u\rangle_{Q_{\varepsilon}^{i} \cap F_{1}^{\varepsilon}}\right| & =\left|\sum_{i=1}^{K_{\varepsilon}}\left(\langle u\rangle_{Q_{\varepsilon}^{i} \cap F_{1}^{\varepsilon}}-\langle u\rangle_{Q_{\varepsilon}^{i}}\right)\right| \\
& \leqslant \sum_{i=1}^{K_{\varepsilon}}\left|\langle u\rangle_{Q_{\varepsilon}^{i} \cap F_{1}^{\varepsilon}}-\langle u\rangle_{Q_{\varepsilon}^{i}}\right| \leqslant\left(K_{\varepsilon} \sum_{i=1}^{K_{\varepsilon}}\left|\langle u\rangle_{Q_{\varepsilon}^{i} \cap F_{1}^{\varepsilon}}-\langle u\rangle_{Q_{\varepsilon}^{i}}\right|^{2}\right)^{1 / 2} .
\end{aligned}
$$

Using inequality (3.17), we can make a further estimate of (3.18) to obtain

$$
\left|\sum_{i=1}^{K_{\varepsilon}}\langle u\rangle_{Q_{\varepsilon}^{i} \cap F_{1}^{\varepsilon}}\right| \leqslant C_{2}\left(K_{\varepsilon} \varepsilon^{2-d}\right)^{1 / 2}\|\nabla u\|_{L^{2}(\boldsymbol{T})} .
$$

${ }^{11}$ Note that, in spite of the assumptions $\mathfrak{f}_{0} \neq 0, \mathfrak{f}_{1} \neq 0$ in lemma 3.2 , we can drop the first of them in the course of getting the estimates (3.12)-(3.15), for, clearly, if $\mathfrak{f}_{0}=0$, then $\|\bar{u}-\tilde{u}\|_{L^{2}(\boldsymbol{T})}=0$. 
In addition to the estimate (3.19), we claim that there exists a positive constant $\hat{c}$ such that the following inequality holds:

$$
\sum_{i, j=1}^{K_{\varepsilon}}\left|\langle u\rangle_{Q_{\varepsilon}^{i} \cap F_{1}^{\varepsilon}}-\langle u\rangle_{Q_{\varepsilon}^{j} \cap F_{1}^{\varepsilon}}\right|^{2} \leqslant \hat{c} \varepsilon^{-2 d}\|\nabla u\|_{L^{2}\left(\boldsymbol{T} \cap F_{1}^{\varepsilon}\right)}^{2} .
$$

To show this, we use the following particular case of lemma 3.2.

Proposition 3.3. Let $Q$ and $Q^{\prime}$ be two cells that share a common side. There then exists a positive constant $\tilde{c}$, depending only on $F_{1}$, such that, for any function $v \in H^{1}\left(\left(Q \cup Q^{\prime}\right) \cap F_{1}\right)$, the following inequality holds:

$$
\left|\langle v\rangle_{Q \cap F_{1}}-\langle v\rangle_{Q^{\prime} \cap F_{1}}\right| \leqslant \tilde{c}\|\nabla v\|_{L^{2}\left(\left(Q \cup Q^{\prime}\right) \cap F_{1}\right)} .
$$

If $Q_{\varepsilon}^{i}$ and $Q_{\varepsilon}^{j}$ share a common side, then, by rescaling (3.21),

$$
\left|\langle u\rangle_{Q_{\varepsilon}^{i} \cap F_{1}^{\varepsilon}}-\langle u\rangle_{Q_{\varepsilon}^{j} \cap F_{1}^{\varepsilon}}\right|^{2} \leqslant \tilde{c} \varepsilon^{2-d}\left(\|\nabla u\|_{L^{2}\left(Q_{\varepsilon}^{i} \cap F_{1}^{\varepsilon}\right)}^{2}+\|\nabla u\|_{L^{2}\left(Q_{\varepsilon}^{j} \cap F_{1}^{\varepsilon}\right)}^{2}\right) .
$$

Let $\left(m_{k}^{1}, m_{k}^{2}, \ldots, m_{k}^{d}\right)$ be the $d$-tuple of coordinates of the centre of the cell $Q_{\varepsilon}^{k}$, $k=1, \ldots, K_{\varepsilon}$. We fix two arbitrary cells $Q_{\varepsilon}^{i}$ and $Q_{\varepsilon}^{j}$ and construct a 'path' across consecutive cells in the cube $\boldsymbol{T}$, such that $Q_{\varepsilon}^{i}$ and $Q_{\varepsilon}^{j}$ are its 'endpoints', in the following way. First, we move along the segment $x_{k}(t)=m_{i}^{1}+t\left(m_{j}^{1}-m_{i}^{1}\right), t \in$ $[0,1]$, so that only the first coordinate of cells changes; the others are fixed and equal to those of the starting endpoint. Upon reaching the cell $\left(m_{j}^{1}, m_{i}^{2}, \ldots, m_{i}^{d}\right)$, we change the direction of the path so that, at the second leg of the path, only the second coordinate changes. Upon reaching the cell $\left(m_{j}^{1}, m_{j}^{2}, \ldots, m_{i}^{d}\right)$, we 'turn' again, and so on, until we reach the cell $\left(m_{j}^{1}, m_{j}^{2}, \ldots, m_{j}^{d}\right)$. Let us now number the cells that are members of the constructed path in the order of passing them, from 1 to $M_{i j}$, where $M_{i j}$ is the total number of cells involved in the path, so that cell $Q_{\varepsilon}^{i}$ has number 1 and cell $Q_{\varepsilon}^{j}$ has number $M_{i j}$. Obviously, $M_{i j} \leqslant 2 d N$, where $N=\varepsilon^{-1} T$. Let us introduce a temporary notation $Q_{r}$ for the cell with number $r$ in the path, $r=1, \ldots, M_{i j}$. It follows from inequality (3.22) that

$$
\begin{aligned}
\left|\langle u\rangle_{Q_{\varepsilon}^{i} \cap F_{1}^{\varepsilon}}-\langle u\rangle_{Q_{\varepsilon}^{j} \cap F_{1}^{\varepsilon}}\right|^{2} & =\left|\sum_{r=1}^{M_{i j}-1}\left(\langle u\rangle_{Q_{r}}-\langle u\rangle_{Q_{r+1}}\right)\right|^{2} \\
& \leqslant\left(M_{i j}-1\right) \sum_{r=1}^{M_{i j}-1}\left|\langle u\rangle_{Q_{r}}-\langle u\rangle_{Q_{r+1}}\right|^{2} \\
& \leqslant\left(M_{i j}-1\right) \tilde{c} \varepsilon^{2-d} \sum_{r=1}^{M_{i j}-1}\left(\|\nabla u\|_{L^{2}\left(Q_{r}\right)}^{2}+\|\nabla u\|_{L^{2}\left(Q_{r+1}\right)}^{2}\right) \\
& \leqslant 4 d N \tilde{c} \varepsilon^{2-d} \sum_{r=1}^{M_{i j}}\|\nabla u\|_{L^{2}\left(Q_{r}\right)}^{2}
\end{aligned}
$$

We perform the above procedure for any pair $(i, j)$ of endpoints. It is not difficult to see that the total number of times that any particular cell is encountered during this process does not exceed $d(2 N)^{d+1}$. Therefore, summing (3.23) over all possible $i, j$, 
we get

$$
\begin{aligned}
\sum_{i, j=1}^{K_{\varepsilon}}\left|\langle u\rangle_{Q_{\varepsilon}^{i} \cap F_{1}^{\varepsilon}}-\langle u\rangle_{Q_{\varepsilon}^{j} \cap F_{1}^{\varepsilon}}\right|^{2} & \leqslant 2^{d+3} d^{2} N^{d+2} \tilde{c} \varepsilon^{2-d}\|\nabla u\|_{L^{2}(\boldsymbol{T})}^{2} \\
& =2^{d+3} d^{2} T^{d+2} \tilde{c} \varepsilon^{-2 d}\|\nabla u\|_{L^{2}(\boldsymbol{T})}^{2}
\end{aligned}
$$

Hence, it is proved that (3.20) holds with $\hat{c}=2^{d+3} d^{2} T^{d+2} \tilde{c}$. Using inequalities (3.19) and (3.20), we can now complete estimation of the third term in the right-hand side of $(3.5)$ :

$$
\begin{aligned}
\|\tilde{u}\|_{L^{2}(\boldsymbol{T})}^{2} & =\varepsilon^{d} \sum_{i}\langle u\rangle_{Q_{\varepsilon}^{i} \cap F_{1}^{\varepsilon}}^{2} \\
& =\varepsilon^{d}\left(2 K_{\varepsilon}\right)^{-1}\left(\sum_{i, j}\left|\langle u\rangle_{Q_{\varepsilon}^{i} \cap F_{1}^{\varepsilon}}-\langle u\rangle_{Q_{\varepsilon}^{j} \cap F_{1}^{\varepsilon}}\right|^{2}+2\left(\sum_{i}\langle u\rangle_{Q_{\varepsilon}^{i} \cap F_{1}^{\varepsilon}}\right)^{2}\right) \\
& \leqslant \varepsilon^{d}\left(2 K_{\varepsilon}\right)^{-1} \hat{c} \varepsilon^{-2 d}\|\nabla u\|_{L^{2}\left(\boldsymbol{T} \cap F_{1}^{\varepsilon}\right)}^{2}+\varepsilon^{d}\left(2 K_{\varepsilon}\right)^{-1} C_{2}^{2} 2 K_{\varepsilon} \varepsilon^{2-d}\|\nabla u\|_{L^{2}(\boldsymbol{T})}^{2} \\
& =\tilde{C}^{2}\|\nabla u\|_{L^{2}\left(\boldsymbol{T} \cap F_{1}^{\varepsilon}\right)}^{2}+C_{2}^{2} \varepsilon^{2}\|\nabla u\|_{L^{2}(\boldsymbol{T})}^{2},
\end{aligned}
$$

where $\tilde{C}=2 T d \sqrt{\tilde{c}}$, and hence

$$
\|\tilde{u}\|_{L^{2}(\boldsymbol{T})} \leqslant \tilde{C}\|\nabla u\|_{L^{2}\left(\boldsymbol{T} \cap F_{1}^{\varepsilon}\right)}+C_{2} \varepsilon\|\nabla u\|_{L^{2}(\boldsymbol{T})}^{2} .
$$

Finally, by summing inequalities (3.6), (3.16) and (3.24) we get the required inequality (3.1) with $C=\max \left\{C_{1}+2 C_{2}, \tilde{C}\right\}$.

The availability of the Poincaré-type inequality (3.1) allows us to apply the argument of $\S 2.2$ to the problem (2.2) with $\lambda=0$, subject to the appropriate restrictions on the averages of the right-hand side $f(\boldsymbol{x})$ and solution $u^{\varepsilon}(\boldsymbol{x})$ (see the text following (2.2)), and under the requirement that the boundary of the set $F_{0}$ be Lipschitz continuous. Indeed, even under the assumption that $\lambda=0$ in (2.13), the compactness property for the 'sequences' $u^{\varepsilon}(\boldsymbol{x})$ and $\varepsilon \nabla u^{\varepsilon}(\boldsymbol{x})$ persists, in view of (3.1). Further, by extracting a (weakly) two-scale convergent subsequence and passing to the limit in the identity $(2.3)$, we arrive at (2.24) with $\lambda=0$, or, equivalently, (2.26), (2.27) with $\lambda=0$.

We next demonstrate that the problem (2.26), (2.27) leads to a (non-local) integro-differential equation on the limiting function $u^{(1)}(\boldsymbol{x})$.

\section{Non-local nature of the homogenized system (2.26)}

Now we intend to show how non-locality arises from consideration of the homogenized system (2.26), where we set $\lambda=0$ and assume that the boundary $\partial F_{0}$ is Lipschitz continuous. Before doing this we make one more remark. Note that the problem $(2.26),(2.27)$ is understood in the weak sense, i.e. in the sense of the identity (2.24). If the right-hand side $f(\boldsymbol{x})$ of the system belongs to the space $L^{2}(\boldsymbol{T})$, as it has so far, we can a priori claim that the solution $\left(u^{(1)}, w\right)$ belongs to the space $H_{\text {per }}^{1}(\boldsymbol{T}) \times L^{2}\left(\boldsymbol{T}, H_{\text {per }}^{1}(Q)\right)$. However, if the right-hand side $f(\boldsymbol{x})$ of the original equation is smooth, then the function $u^{(1)}(\boldsymbol{x})$ representing the restriction of the 
homogenized solution to the hard phase is smooth too. This fact is obtained as a by-product in what follows.

We denote by

$$
G=G\left(y_{1}, y_{2}, y_{1}^{\prime}, y_{2}^{\prime}, x_{3}\right)
$$

the Green function of the operator $-\partial_{y_{1}}^{2}-\partial_{y_{2}}^{2}-\alpha \partial_{x_{3}}^{2}$ in the cylinder $\left(\tilde{F}_{0} \cap Q_{2}\right) \times$ $[-T, T]$ with periodic boundary condition on the 'bases' $x_{3}= \pm T$, Dirichlet conditions on $\left(\partial \tilde{F}_{0} \cap Q_{2}\right) \times[-T, T]$, and $Q_{2}$-periodicity conditions on $\operatorname{Int}\left(\tilde{F}_{0}\right) \cap \partial Q_{2}$, where $\operatorname{Int}\left(\tilde{F}_{0}\right)$ denotes the interior of $\tilde{F}_{0}$. The above Green function exists (see, for example, [25]). From the second equation in system $(2.26)$, where $\lambda=0$, we get

$$
w(\boldsymbol{x}, \tilde{\boldsymbol{y}})=\mathfrak{f}_{0} \int_{-T}^{T}\left\langle G\left(\tilde{\boldsymbol{y}}, \tilde{\boldsymbol{y}}^{\prime}, x_{3}-x_{3}^{\prime}\right)\right\rangle_{\tilde{y}^{\prime}}\left(\alpha^{-1} f\left(\tilde{\boldsymbol{x}}^{\prime}, x_{3}^{\prime}\right)+u_{, x_{3}^{\prime} x_{3}^{\prime}}^{(1)}\left(\tilde{\boldsymbol{x}}, x_{3}^{\prime}\right)\right) \mathrm{d} x_{3}^{\prime},
$$

where $\left\langle G\left(\tilde{\boldsymbol{y}}, \tilde{\boldsymbol{y}}^{\prime}, x_{3}-x_{3}^{\prime}\right)\right\rangle_{\tilde{y}^{\prime}}$ is the average of the Green function with respect to the vector $\tilde{\boldsymbol{y}}^{\prime} \in \tilde{F}_{0} \cap Q_{2}$. We write (4.1) also in a concise form as follows:

$$
w=\mathfrak{f}_{0}\langle G\rangle_{\tilde{y}^{\prime}} \stackrel{x_{3}}{*}\left(\alpha^{-1} f+u_{, x_{3} x_{3}}^{(1)}\right),
$$

where the symbol $\stackrel{x_{3}}{*}$, denotes convolution with respect to the variable $x_{3}$ only.

Substituting expression (4.2) for the function $w(\boldsymbol{x}, \tilde{\boldsymbol{y}})$ into the first equation of the system $(2.26)$, we obtain

$$
-\operatorname{div}\left(A^{\mathrm{hom}} \nabla u^{(1)}\right)-\alpha \mathfrak{f}_{0}^{2}\langle G\rangle_{\tilde{y}^{\prime}, \tilde{y}} \stackrel{x_{3}}{*} \frac{\partial^{4} u^{(1)}}{\partial x_{3}^{4}}=f+\mathfrak{f}_{0}^{2}\langle G\rangle_{\tilde{y}^{\prime}, \tilde{y}} \stackrel{x_{3}}{*} \frac{\partial^{2} f}{\partial x_{3}^{2}} .
$$

To prove that a periodic solution to the equation (4.3) is infinitely smooth we use some standard techniques of Fourier analysis. To this end observe that, if we denote

$$
F:=f+\mathfrak{f}_{0}^{2}\langle G\rangle_{\tilde{y}^{\prime}, \tilde{y}} \stackrel{x_{3}}{*} \frac{\partial^{2} f}{\partial x_{3}^{2}},
$$

then the Fourier coefficients $\hat{u}^{(1)}(\boldsymbol{m})$ and $\hat{F}(\boldsymbol{m}), \boldsymbol{m} \in \mathbb{Z}^{3}$, of the periodic functions $u^{(1)}(\boldsymbol{x})$ and $F(\boldsymbol{x})$ are related by the following formula (henceforth in this section we assume, without loss of generality, that $T=\pi)$ :

$$
\left(\tilde{h}_{p r} m_{p} m_{r}+\left(\mathfrak{f}_{1}+\alpha \mathfrak{f}_{0}\right) m_{3}^{2}-\alpha \mathfrak{f}_{0}^{2} m_{3}^{4}\left\langle\hat{\mathcal{K}}\left(\tilde{\boldsymbol{y}}, m_{3}\right)\right\rangle_{\tilde{y}}\right) \hat{u}^{(1)}(\boldsymbol{m})=\hat{F}(\boldsymbol{m}) .
$$

Here

$$
\tilde{h}_{i j}:=\int_{\tilde{F}_{1} \cap Q_{2}}\left(\delta_{i j}+\left(N_{i}\right)_{, j}(\tilde{\boldsymbol{y}})\right) \mathrm{d} \tilde{\boldsymbol{y}}, \quad i, j=1,2,
$$

are elements of the matrix $A_{2 \mathrm{D}}^{\text {hom }}($ see $(2.7))$, and $\hat{\mathcal{K}}\left(\tilde{\boldsymbol{y}}, m_{3}\right)$ are the Fourier coefficients of the function $\mathcal{K}\left(\tilde{\boldsymbol{y}}, x_{3}\right):=\left\langle G\left(\tilde{\boldsymbol{y}}, \tilde{\boldsymbol{y}}^{\prime}, x_{3}\right)\right\rangle_{\tilde{y}^{\prime}}$. Note that the Green function $G$ is the $x_{3}$-periodic solution to the following boundary-value problem (henceforth we also assume the $Q_{2}$-periodicity condition on $\operatorname{Int}\left(\tilde{F}_{0}\right) \cap \partial Q_{2}$ without mentioning it explicitly):

$$
\begin{gathered}
-\frac{\partial^{2} G}{\partial y_{1}^{2}}-\frac{\partial^{2} G}{\partial y_{2}^{2}}-\frac{\partial^{2} G}{\partial x_{3}^{2}}=\delta\left(\tilde{\boldsymbol{y}}-\tilde{\boldsymbol{y}}^{\prime}, x_{3}\right), \quad\left(\tilde{\boldsymbol{y}}, x_{3}\right),\left(\tilde{\boldsymbol{y}}^{\prime}, x_{3}\right) \in\left(\tilde{F}_{0} \cap Q_{2}\right) \times[-T, T] \\
\left.G\left(\tilde{\boldsymbol{y}}, \tilde{\boldsymbol{y}}^{\prime}, x_{3}\right)\right|_{\tilde{y} \in \partial \tilde{F}_{0} \cap Q_{2}}=0 .
\end{gathered}
$$


Taking the average with respect to $\tilde{\boldsymbol{y}}^{\prime} \in \tilde{F}_{0} \cap Q_{2}$ in (4.5), (4.6), we arrive at a boundary-value problem for the function $\mathcal{K}(\tilde{\boldsymbol{y}})$ as follows:

$$
\begin{gathered}
-\frac{\partial^{2} \mathcal{K}}{\partial y_{1}^{2}}-\frac{\partial^{2} \mathcal{K}}{\partial y_{2}^{2}}-\frac{\partial^{2} \mathcal{K}}{\partial x_{3}^{2}}=\mathfrak{f}_{0}^{-1} \delta\left(x_{3}\right), \quad\left(\tilde{\boldsymbol{y}}, x_{3}\right) \in\left(\tilde{F}_{0} \cap Q_{2}\right) \times[-T, T], \\
\left.\mathcal{K}\left(\tilde{\boldsymbol{y}}, x_{3}\right)\right|_{\tilde{y} \in \partial \tilde{F}_{0} \cap Q_{2}}=0 .
\end{gathered}
$$

Hence, the following boundary-value problem for the Fourier coefficients $\hat{\mathcal{K}}\left(\tilde{\boldsymbol{y}}, m_{3}\right)$ holds:

$$
\begin{gathered}
-\frac{\partial^{2} \hat{\mathcal{K}}\left(\tilde{\boldsymbol{y}}, m_{3}\right)}{\partial y_{1}^{2}}-\frac{\partial^{2} \hat{\mathcal{K}}\left(\tilde{\boldsymbol{y}}, m_{3}\right)}{\partial y_{2}^{2}}+m_{3}^{2} \hat{\mathcal{K}}\left(\tilde{\boldsymbol{y}}, m_{3}\right)=\mathfrak{f}_{0}^{-1}, \quad \tilde{\boldsymbol{y}} \in \tilde{F}_{0} \cap Q_{2}, \\
\left.\hat{\mathcal{K}}\left(\tilde{\boldsymbol{y}}, m_{3}\right)\right|_{\tilde{\boldsymbol{y}} \in \partial \tilde{F}_{0} \cap Q_{2}}=0 .
\end{gathered}
$$

We treat $m_{3} \in \mathbb{Z}$ in (4.7)-(4.8) as a parameter. Clearly,

$$
\hat{\mathcal{K}}\left(\tilde{\boldsymbol{y}}, m_{3}\right)=\mathfrak{f}_{0}^{-1} \int_{\tilde{F}_{0} \cap Q_{2}} \mathcal{G}\left(\tilde{\boldsymbol{y}}, \tilde{\boldsymbol{y}}^{\prime}, m_{3}\right) \mathrm{d} \tilde{\boldsymbol{y}}^{\prime},
$$

where $\mathcal{G}\left(\tilde{\boldsymbol{y}}, \tilde{\boldsymbol{y}}^{\prime}, m_{3}\right)$ is the Green function of the operator $-\partial_{y_{1}}^{2}-\partial_{y_{2}}^{2}+m_{3}^{2}$ in the domain $\tilde{F}_{0} \cap Q_{2}$. The formula (4.9) implies that

$$
\left\langle\hat{\mathcal{K}}\left(\tilde{\boldsymbol{y}}, m_{3}\right)\right\rangle_{\tilde{y}}=\mathfrak{f}_{0}^{-2} \int_{\tilde{F}_{0} \cap Q_{2}} \int_{\tilde{F}_{0} \cap Q_{2}} \mathcal{G}\left(\tilde{\boldsymbol{y}}, \tilde{\boldsymbol{y}}^{\prime}, m_{3}\right) \mathrm{d} \tilde{\boldsymbol{y}}^{\prime} \mathrm{d} \tilde{\boldsymbol{y}} .
$$

The Green function $\mathcal{G}$ can be split into two parts: the fundamental solution $\mathcal{E}$ of the operator $-\partial_{y_{1}}^{2}-\partial_{y_{2}}^{2}+m_{3}^{2}$ in $\mathbb{R}^{2}$ and the 'reflected' part $\tilde{\mathcal{G}}$ as follows:

$$
\mathcal{G}\left(\tilde{\boldsymbol{y}}, \tilde{\boldsymbol{y}}^{\prime}, m_{3}\right)=\mathcal{E}\left(\tilde{\boldsymbol{y}}-\tilde{\boldsymbol{y}}^{\prime}, m_{3}\right)+\tilde{\mathcal{G}}\left(\tilde{\boldsymbol{y}}, \tilde{\boldsymbol{y}}^{\prime}, m_{3}\right) .
$$

The fundamental solution $\mathcal{E}$ is well known, namely,

$$
\mathcal{E}\left(\boldsymbol{z}, m_{3}\right)=\frac{1}{2 \pi} K_{0}\left(\left|m_{3}\right||\boldsymbol{z}|\right), \quad z \in \mathbb{R}^{2},
$$

where $m_{3} \neq 0$ and $K_{0}$ is a modified Bessel function of the third kind.

Due to formula (4.11), the value $\left\langle\hat{\mathcal{K}}\left(\tilde{\boldsymbol{y}}, m_{3}\right)\right\rangle_{\tilde{y}}$ in $(4.10)$ splits into two corresponding parts as follows:

$$
\begin{aligned}
\left\langle\hat{\mathcal{K}}\left(\tilde{\boldsymbol{y}}, m_{3}\right)\right\rangle_{\tilde{y}}=\mathfrak{f}_{0}^{-2} \int_{\tilde{F}_{0} \cap Q_{2}} \int_{\tilde{F}_{0} \cap Q_{2}} \mathcal{E}\left(\tilde{\boldsymbol{y}}-\tilde{\boldsymbol{y}}^{\prime}, m_{3}\right) \mathrm{d} \tilde{\boldsymbol{y}}^{\prime} \mathrm{d} \tilde{\boldsymbol{y}} \\
\quad+\mathfrak{f}_{0}^{-2} \int_{\tilde{F}_{0} \cap Q_{2}} \int_{\tilde{F}_{0} \cap Q_{2}} \tilde{\mathcal{G}}\left(\tilde{\boldsymbol{y}}, \tilde{\boldsymbol{y}}^{\prime}, m_{3}\right) \mathrm{d} \tilde{\boldsymbol{y}}^{\prime} \mathrm{d} \tilde{\boldsymbol{y}} .
\end{aligned}
$$

It is relatively easy to estimate the first term in the right-hand side of (4.13). To this end, note that, using formula (4.12) and assuming henceforth that $m_{3} \neq 0$, we 
obtain

$$
\begin{aligned}
\mathfrak{f}_{0}^{-2} \int_{\tilde{F}_{0} \cap Q_{2}} & \int_{\tilde{F}_{0} \cap Q_{2}} \mathcal{E}\left(\tilde{\boldsymbol{y}}-\tilde{\boldsymbol{y}}^{\prime}, m_{3}\right) \mathrm{d} \tilde{\boldsymbol{y}}^{\prime} \mathrm{d} \tilde{\boldsymbol{y}} \\
& \leqslant \mathfrak{f}_{0}^{-2} \int_{\tilde{F}_{0} \cap Q_{2}} \int_{R^{2}} \mathcal{E}\left(\tilde{\boldsymbol{y}}-\tilde{\boldsymbol{y}}^{\prime}, m_{3}\right) \mathrm{d} \tilde{\boldsymbol{y}}^{\prime} \mathrm{d} \tilde{\boldsymbol{y}} \\
& =\mathfrak{f}_{0}^{-1} \int_{\tilde{F}_{0} \cap Q_{2}} \int_{R^{2}} \mathcal{E}\left(\tilde{\boldsymbol{z}}, m_{3}\right) \mathrm{d} \tilde{\boldsymbol{z}} \mathrm{d} \tilde{\boldsymbol{y}}^{\prime} \\
& =\frac{\mathfrak{f}_{0}^{-1}}{2 \pi} \int_{R^{2}} K_{0}\left(\left|m_{3}\right||\tilde{\boldsymbol{z}}|\right) \mathrm{d} \tilde{\boldsymbol{z}}=\mathfrak{f}_{0}^{-1} m_{3}^{-2} \int_{0}^{\infty} K_{0}(\zeta) \zeta \mathrm{d} \zeta=\mathfrak{f}_{0}^{-1} m_{3}^{-2} .
\end{aligned}
$$

The last equality results from the following formula:

$$
\int_{0}^{\infty} K_{0}(\zeta) \zeta \mathrm{d} \zeta=1
$$

(see, for example, [14, formula 6.561(16), p. 668]).

The function $\tilde{\mathcal{G}}$ is non-positive, as follows from the maximum principle. Hence, recalling the equation (4.4) for the Fourier coefficients of the function $u^{(1)}(\boldsymbol{x})$, we get

$$
\hat{u}^{(1)}(\boldsymbol{m})=(V(\boldsymbol{m}))^{-1} \hat{F}(\boldsymbol{m}),
$$

where

$$
\begin{aligned}
V(\boldsymbol{m}) & :=\tilde{h}_{p r} m_{p} m_{r}+\left(\mathfrak{f}_{1}+\alpha \mathfrak{f}_{0}\right) m_{3}^{2}-\alpha \mathfrak{f}_{0}^{2} m_{3}^{4}\left\langle\hat{\mathcal{K}}\left(\tilde{\boldsymbol{y}}, m_{3}\right)\right\rangle_{\tilde{y}} \\
& \geqslant \tilde{h}_{p r} m_{p} m_{r}+\mathfrak{f}_{1} m_{3}^{2} .
\end{aligned}
$$

Due to the fact that the function $F(\boldsymbol{x})$ is infinitely smooth, its Fourier coefficients decay faster than any power of $|\boldsymbol{m}|$ when $|\boldsymbol{m}| \rightarrow \infty$. Therefore, in view of formula (4.14) and the estimate (4.15), the Fourier coefficients of the function $u^{(1)}(\boldsymbol{x})$ also decay faster than any power of $|\boldsymbol{m}|$, which implies smoothness of the function $u^{(1)}(\boldsymbol{x})$. Hence, we have proved the following theorem.

TheOrem 4.1. Let $\lambda=0$ in equations (2.2), $f(\boldsymbol{x}) \in C_{0}^{\infty}(\boldsymbol{T})$,

$$
\int_{\boldsymbol{T}} f(\boldsymbol{x}) \mathrm{d} \boldsymbol{x}=0
$$

and the boundary of the set $F_{0}$ is Lipschitz continuous. Then the restriction $u^{(1)}(\boldsymbol{x})$ of the two-scale limit of the solutions $u^{\varepsilon}(\boldsymbol{x})$ to the hard phase $F_{1}$ is infinitely smooth and satisfies the integro-differential equation (4.3).

The above result establishes that the limit $u^{(1)}(\boldsymbol{x})$ of the restriction of $u^{\varepsilon}(\boldsymbol{x})$ on the matrix solves the non-local equation (4.3), and in this sense (4.3) may be regarded as a non-local homogenized limit of the original equation (2.2).

\section{Discussion}

In this work we proposed a study of a linear periodic rapidly oscillating problem set on a mixture of materials whose homogenized limit exhibits a spatially nonlocal behaviour. The problem derives from an equation with a scaling of 'double 
porosity' type, which has previously been mathematically investigated by a number of authors (see, for example, $[1,28]$ and further references therein). The key feature of the double porosity models is that the small parameter $\varkappa$ of contrast between the constituent phases and the small period $\varepsilon$ of the coefficients of the original heterogeneous equation are related by the formula $\varkappa \sim \varepsilon^{2}$. It has been demonstrated in a number of settings (see also $[6,20]$ ) that problems of this type may lead to non-locality in the overall behaviour. However, the case of high anisotropy in the properties of one of the phases (with the related 'anisotropy scaling' of the double porosity type) remained unaddressed.

Using these ideas as a starting point, we studied a model situation of a mixture of two conducting materials, one of which is included in the other as periodic fibres having conductivity of order 1 in the direction along the fibres and very small conductivities (of order $\varepsilon^{2}$ ) in the directions orthogonal to the fibres. We demonstrated in theorem 2.2 how a version of the two-scale convergence method, widely used in the literature over the recent years for passing to the limit in rapidly oscillating problems, can be used to find the non-local homogenized limit and to adequately describe the convergence of solutions.

These results were first obtained under the restriction $\lambda>0$ on the 'spectral parameter' $\lambda$ in the original problem (2.2). However, we further showed that the case $\lambda=0$ can also be accounted for, via the use of a 'non-standard' version of the Poincaré inequality (see theorem 3.1). The homogenized limit in the matrix $u^{(1)}(\boldsymbol{x})$ is shown to satisfy a convolution-type homogenized equation (theorem 4.1), thus exhibiting non-locality in the overall behaviour.

As discussed at the end of $\S 2.1$, the results stated in this paper could be extended in a rather straightforward way to a slightly more general case of high anisotropy (see (2.9) and (2.10)).

The results presented may also be of interest as precedents for mathematical study of a number of interesting phenomena. We next outline two of these.

First, the above non-locality statement, rigorously established using the technique of two-scale convergence, can also be re-derived formally, from the straingradient asymptotics (see $[9,11,21]$ ) of the solution to the (uniformly elliptic) problem $-\left(A_{i j}^{\varkappa}(\boldsymbol{x} / \varepsilon) u_{, j}\right)_{, i}=f(\boldsymbol{x})$, where the matrix of coefficients is initially determined by the formula $\left(A_{i j}^{\varkappa}(\boldsymbol{y})\right)=\operatorname{diag}(\varkappa, \varkappa, 1)$ if $\boldsymbol{y} \in F_{0}$, and by $\left(A_{i j}^{\varkappa}(\boldsymbol{y})\right)=\operatorname{diag}(1,1,1)$ if $\boldsymbol{y} \in F_{1}$. Firstly, fixing $\varkappa$ and treating $\varepsilon$ as a parameter, we arrive at a twoscale asymptotic expansion of the kind discussed in $[9,11,21]$, with coefficients depending on $\varkappa$. Further, one can observe that when $\varkappa$ is of order $\varepsilon^{2}$ this asymptotic expansion 'breaks up': all the terms become of equal 'strength'. It turns out that the main-order terms constitute certain expansion to the two-scale limit $u_{0}(\boldsymbol{x})+w\left(\boldsymbol{x}, x_{1} / \varepsilon, x_{2} / \varepsilon\right)$ (see [9, ch. 4] for more details). This observation may point at certain relations between the so-called 'size effects' widely documented in engineering literature (see, for example, [13]) and the non-locality effects in the overall response of periodic media when expressed in terms of 'ensemble mean' stresses and strains for the underlying 'small but fixed' $\varepsilon$ (see, for example, [26]). The doubleporosity models (for which $\varkappa \sim \varepsilon^{2}$ ) are therefore a good testing ground for exploring a relationship between these phenomena, since the overall constitutive laws for such models are non-local not only for fixed $\varepsilon>0$ but also in the homogenized limit as $\varepsilon \rightarrow 0$, a feature not present in the 'classical' uniformly elliptic homogenization. 
Secondly, in the context of wave propagation, in both acoustics and electromagnetism, the property of 'intrinsic non-locality' of the double-porosity models may be related to the "propagation versus localization" effects in high-contrast media. The dependence of the overall response at a given point on values of the fields at 'remote' points in the present static setting may be analogous in the dynamic setting of wave propagation to the capability of the medium to guide oscillations with certain frequencies along the fibres, while 'forbidding' their transverse spreading. There has been an explosion of interest in these phenomena among physicists and experimentalists concerned with physical properties of photonic and phononic crystals for fibre optics and for optimal design of elastics waveguides (see, for example, [22]). The latter has also been gaining interest in the mathematical community (see, for example, [17]): a tendency that is certain to grow in view of the scope of potential applications.

\section{Acknowledgments}

The authors acknowledge an interesting comment by an anonymous referee towards the possibility of a generalization of the results presented in this paper, which led us to $(2.9),(2.10)$.

\section{Appendix A. Two-scale convergence: definition and basic properties}

Here we review some of the facts about the two-scale convergence, due to Nguetseng [18], Allaire [1] and Zhikov [28]. (Some properties are formulated in a more general setting involving periodic measures (see [28]).)

1. Definition. Let $\mu$ be a $Q$-periodic Borel measure in $\mathbb{R}^{d}, \boldsymbol{T}$ a bounded domain in $\mathbb{R}^{d}$. Define the 'scaling' measure $\mu^{\varepsilon}$ by setting $\mu^{\varepsilon}=\varepsilon^{d} \mu\left(\varepsilon^{-1} B\right)$ for any Borel set $B \subset \mathbb{R}^{d}$. A sequence of functions $u^{\varepsilon}(\boldsymbol{x}) \in L^{2}\left(\boldsymbol{T}, \mathrm{d} \mu^{\varepsilon}\right)$ is said to (weakly) twoscale converge to the function $u(\boldsymbol{x}, \boldsymbol{y}) \in L^{2}(\boldsymbol{T} \times Q, \mathrm{~d} \boldsymbol{x} \times \mathrm{d} \mu)$ (with respect to the measure $\mu$ ), if for any test function $\psi(\boldsymbol{x}, \boldsymbol{y}) \in C(\boldsymbol{T} \times Q)$ :

$$
\int_{\boldsymbol{T}} u^{\varepsilon}(\boldsymbol{x}) \psi\left(\boldsymbol{x}, \frac{\boldsymbol{x}}{\varepsilon}\right) \mathrm{d} \mu^{\varepsilon} \stackrel{\varepsilon \rightarrow 0}{\longrightarrow} \int_{\boldsymbol{T}} \int_{Q} u(\boldsymbol{x}, \boldsymbol{y}) \psi(\boldsymbol{x}, \boldsymbol{y}) \mathrm{d} \mu(\boldsymbol{y}) \mathrm{d} \boldsymbol{x} .
$$

If $u^{\varepsilon}(\boldsymbol{x})$ two-scale converges to $u(\boldsymbol{x}, \boldsymbol{y})$, we write $u^{\varepsilon}(\boldsymbol{x}) \stackrel{2}{\rightarrow} u(\boldsymbol{x}, \boldsymbol{y})$, assuming that it is clear from the context what measure is associated with the convergence.

2. Compactness property. If a sequence of functions $u^{\varepsilon}(\boldsymbol{x}) \in L^{2}\left(\boldsymbol{T}, \mathrm{d} \mu^{\varepsilon}\right)$ is bounded in $L^{2}\left(\boldsymbol{T}, \mathrm{d} \mu^{\varepsilon}\right)$, then there exist a subsequence $u^{\varepsilon_{j}}(\boldsymbol{x})$ and a function

$$
u(\boldsymbol{x}, \boldsymbol{y}) \in L^{2}(\boldsymbol{T} \times Q, \mathrm{~d} \boldsymbol{x} \times \mathrm{d} \mu)
$$

(possibly depending on the chosen subsequence), such that $u^{\varepsilon_{j}}(\boldsymbol{x}) \stackrel{2}{\rightarrow} u(\boldsymbol{x}, \boldsymbol{y})$.

3. If the sequences $u^{\varepsilon}(\boldsymbol{x}) \in H^{1}(\boldsymbol{T})$ and $\varepsilon \nabla u^{\varepsilon}(\boldsymbol{x})$ are bounded in $L^{2}(\boldsymbol{T})$ and $\left[L^{2}(\boldsymbol{T})\right]^{3}$, respectively, then there exists a function $u(\boldsymbol{x}, \boldsymbol{y}) \in L^{2}\left(\boldsymbol{T}, H_{\mathrm{per}}^{1}(Q)\right)$ such that, up to a subsequence, $u^{\varepsilon}(\boldsymbol{x}) \stackrel{2}{\rightarrow} u(\boldsymbol{x}, \boldsymbol{y})$ and $\varepsilon \nabla u^{\varepsilon}(\boldsymbol{x}) \stackrel{2}{\rightarrow} \nabla_{y} u(\boldsymbol{x}, \boldsymbol{y})$ with respect to the Lebesgue measure on $Q$. 
4. Multiplication by a bounded function. If $u^{\varepsilon}(\boldsymbol{x}) \stackrel{2}{\rightarrow} u(\boldsymbol{x}, \boldsymbol{y})$ and $\varphi(\boldsymbol{y}) \in L^{\infty}(Q)$, then

$$
u^{\varepsilon}(\boldsymbol{x}) \varphi\left(\frac{\boldsymbol{x}}{\varepsilon}\right) \stackrel{2}{\rightarrow} u(\boldsymbol{x}, \boldsymbol{y}) \varphi(\boldsymbol{y}) .
$$

5. Relation to weak convergence. If $u^{\varepsilon}(\boldsymbol{x}) \stackrel{2}{\rightarrow} u(\boldsymbol{x}, \boldsymbol{y})$, then

$$
u^{\varepsilon}(\boldsymbol{x}) \rightarrow \int_{Q} u(\boldsymbol{x}, \boldsymbol{y}) \mathrm{d} \mu(\boldsymbol{y}),
$$

where $\rightarrow$ denotes the usual weak convergence. ${ }^{12}$

\section{Appendix B. The restriction $u^{(1)}(x)$ of the limiting function to the hard phase $u^{(1)}(x)$ belongs to the space $H_{\text {per }}^{1}(T)$}

In this appendix we prove that the 'restriction' $u^{(1)}(\boldsymbol{x})$ of the limiting function $u(\boldsymbol{x}, \boldsymbol{y})$ from $(2.14)$ to the hard phase $F_{1}^{\varepsilon}$, considered as a function of $\boldsymbol{x}$ only, belongs to the Sobolev space $H_{\text {per }}^{1}(\boldsymbol{T})$. The argument follows [28, theorem 4.2].

Denote $\mathrm{d} \mu_{1}^{\varepsilon}:=\left.\mathrm{d} \boldsymbol{x}\right|_{F_{1}^{\varepsilon}}$. Then the relation

$$
\chi_{1}\left(\varepsilon^{-1} \boldsymbol{x}\right) u^{\varepsilon}(\boldsymbol{x}) \stackrel{2}{\rightarrow} \chi_{1}(\boldsymbol{y}) u^{(1)}(\boldsymbol{x}),
$$

which follows from property 4 in Appendix A, can be rewritten in the sense of twoscale convergence with respect to the measure $\mu_{1}$ (see the definition in Appendix A):

$$
L^{2}\left(\boldsymbol{T}, \mathrm{d} \mu_{1}^{\varepsilon}\right) \ni u^{\varepsilon}(\boldsymbol{x}) \stackrel{2}{\rightarrow} u^{(1)}(\boldsymbol{x}) \in L^{2}(\boldsymbol{T}, \mathrm{d} \boldsymbol{x}) .
$$

Note that, for any $\varepsilon>0$, the function $u^{\varepsilon}(\boldsymbol{x})$ is the limit of a sequence of smooth $\boldsymbol{T}$-periodic functions $U_{n}^{\varepsilon}(\boldsymbol{x})$ as $n \rightarrow \infty$ in the $H^{1}(\boldsymbol{T})$-norm. Furthermore, for any function $\phi(\boldsymbol{x}) \in C_{\text {per }}^{\infty}(\boldsymbol{T})$ and vector function $\boldsymbol{h}(\boldsymbol{y}) \in V_{\text {sol }}\left(Q, \mathrm{~d} \mu_{1}\right)^{13}$ and for any $\varepsilon>0, n \in \boldsymbol{N}$, the following formula holds:

$$
\begin{aligned}
\int_{\boldsymbol{T}} \phi(\boldsymbol{x}) & \nabla U_{n}^{\varepsilon}(\boldsymbol{x}) \cdot \boldsymbol{h}\left(\varepsilon^{-1} \boldsymbol{x}\right) \mathrm{d} \mu_{1}^{\varepsilon} \\
& =\int_{\boldsymbol{T}} \nabla\left(U_{n}^{\varepsilon}(\boldsymbol{x}) \phi(\boldsymbol{x})\right) \cdot \boldsymbol{h}\left(\varepsilon^{-1} \boldsymbol{x}\right) \mathrm{d} \mu_{1}^{\varepsilon}-\int_{\boldsymbol{T}} U_{n}^{\varepsilon}(\boldsymbol{x}) \nabla \phi(\boldsymbol{x}) \cdot \boldsymbol{h}\left(\varepsilon^{-1} \boldsymbol{x}\right) \mathrm{d} \mu_{1}^{\varepsilon} \\
& =-\int_{\boldsymbol{T}} U_{n}^{\varepsilon}(\boldsymbol{x}) \nabla \phi(\boldsymbol{x}) \cdot \boldsymbol{h}\left(\varepsilon^{-1} \boldsymbol{x}\right) \mathrm{d} \mu_{1}^{\varepsilon} .
\end{aligned}
$$

Here we have used integration by parts, periodicity of the functions involved and the fact that $\boldsymbol{h}(\boldsymbol{y})$ is a solenoidal vector function. Passing to the limit as $n \rightarrow \infty$, we conclude that

$$
\int_{\boldsymbol{T}} \phi(\boldsymbol{x}) \nabla u^{\varepsilon}(\boldsymbol{x}) \cdot \boldsymbol{h}\left(\varepsilon^{-1} \boldsymbol{x}\right) \mathrm{d} \mu_{1}^{\varepsilon}=-\int_{\boldsymbol{T}} u^{\varepsilon}(\boldsymbol{x}) \nabla \phi(\boldsymbol{x}) \cdot \boldsymbol{h}\left(\varepsilon^{-1} \boldsymbol{x}\right) \mathrm{d} \mu_{1}^{\varepsilon} .
$$

${ }^{12}$ A sequence $u^{\varepsilon}(\boldsymbol{x}) \in L^{2}\left(\boldsymbol{T}, \mathrm{d} \mu^{\varepsilon}\right)$ is said to converge weakly to a function $u(\boldsymbol{x}) \in L^{2}(\boldsymbol{T})$ if, for any test function $\phi(\boldsymbol{x}) \in C(\boldsymbol{T})$, the convergence

$$
\int_{\boldsymbol{T}} u^{\varepsilon}(\boldsymbol{x}) \phi(\boldsymbol{x}) \mathrm{d} \mu^{\varepsilon} \rightarrow \int_{\boldsymbol{T}} u(\boldsymbol{x}) \phi(\boldsymbol{x}) \mathrm{d} \boldsymbol{x}
$$

holds as $\varepsilon \rightarrow 0$.

${ }^{13}$ The set $V_{\text {sol }}\left(Q, \mathrm{~d} \mu_{1}\right)$ of periodic solenoidal vectors (with respect to the measure $\mu_{1}$ ) is defined as the orthogonal complement of the set $\left\{\nabla \phi: \phi \in C_{\text {per }}^{\infty}(Q)\right\}$ in the space $\left[L_{\text {per }}^{2}\left(Q, \mathrm{~d} \mu_{1}\right)\right]^{3}$. 
It follows from the inequality (2.13) that $\nabla u^{\varepsilon}(\boldsymbol{x})$ is bounded in $\left[L^{2}\left(\boldsymbol{T}, \mathrm{d} \mu_{1}^{\varepsilon}\right)\right]^{3}$ and therefore (see the compactness property, Appendix A), there exists a vector function $\boldsymbol{p}(\boldsymbol{x}, \boldsymbol{y}) \in\left[L^{2}\left(\boldsymbol{T} \times Q, \mathrm{~d} \boldsymbol{x} \times \mathrm{d} \mu_{1}\right)\right]^{3}$ such that, up to a subsequence,

$$
\nabla u^{\varepsilon} \stackrel{2}{\rightarrow} \boldsymbol{p}(\boldsymbol{x}, \boldsymbol{y})
$$

Using this and (B1), we pass to the limit in (B 2) and arrive at the following identity:

$$
\int_{\boldsymbol{T}} \int_{Q} \phi(\boldsymbol{x}) \boldsymbol{p}(\boldsymbol{x}, \boldsymbol{y}) \cdot \boldsymbol{h}(\boldsymbol{y}) \mathrm{d} \mu_{1} \mathrm{~d} \boldsymbol{x}=-\int_{\boldsymbol{T}} \int_{Q} u^{(1)}(\boldsymbol{x}) \nabla \phi(\boldsymbol{x}) \cdot \boldsymbol{h}(\boldsymbol{y}) \mathrm{d} \mu_{1} \mathrm{~d} \boldsymbol{x} .
$$

We rewrite the right-hand side of the last formula to get

$$
\int_{\boldsymbol{T}} \int_{Q} \phi(\boldsymbol{x}) \boldsymbol{p}(\boldsymbol{x}, \boldsymbol{y}) \cdot \boldsymbol{h}(\boldsymbol{y}) \mathrm{d} \mu_{1} \mathrm{~d} \boldsymbol{x}=-\int_{\boldsymbol{T}} u^{(1)}(\boldsymbol{x}) \nabla \phi(\boldsymbol{x}) \cdot\langle\boldsymbol{h}\rangle \mathrm{d} \boldsymbol{x} .
$$

Thus, denoting $\langle\boldsymbol{h}\rangle=: \boldsymbol{a} \in \mathbb{R}^{3}$ we conclude that

$$
\boldsymbol{a} \cdot \nabla u^{(1)}(\boldsymbol{x})=\int_{Q} \boldsymbol{p}(\boldsymbol{x}, \boldsymbol{y}) \cdot \boldsymbol{h}(\boldsymbol{y}) \mathrm{d} \mu_{1},
$$

and hence $\boldsymbol{a} \cdot \nabla u^{(1)}(\boldsymbol{x}) \in L^{2}(\boldsymbol{T})$.

From the fact that the measure $\mu_{1}$ is non-degenerate, we deduce that, for any $\boldsymbol{a} \in \mathbb{R}^{3}$, there exists $\boldsymbol{h} \in V_{\text {sol }}\left(Q, \mathrm{~d} \mu_{1}\right)$ such that $\langle\boldsymbol{h}\rangle=\boldsymbol{a}$ (for a proof of this, see [28, proposition 3.2]). Therefore, for any $\boldsymbol{a} \in \mathbb{R}^{3}$, the expression $\boldsymbol{a} \cdot \nabla u^{(1)}(\boldsymbol{x})$ belongs to the Lebesgue class $L^{2}(\boldsymbol{T})$. This results in $\nabla u^{(1)}(\boldsymbol{x}) \in\left[L^{2}(\boldsymbol{T})\right]^{3}$; hence $u^{(1)}(\boldsymbol{x}) \in H^{1}(\boldsymbol{T})$.

Denote by $\mathcal{T}$ the torus obtained in the usual way from the periodicity cell $\boldsymbol{T}$, by identifying appropriate parts of the boundary of $\boldsymbol{T}$. Consider a smooth partition of unity

$$
\sum_{i=1}^{m} \zeta_{m}(\boldsymbol{x})=1 \text { on } \mathcal{T}
$$

such that, for any $i=1, \ldots, m, \operatorname{supp}\left(\zeta_{i}\right)$ is contained in an appropriately 'translated' copy, $\boldsymbol{T}_{i}$, of $\boldsymbol{T}$. Then, repeating the above argument for the sequence $u^{\varepsilon}(\boldsymbol{x}) \zeta_{i}(\boldsymbol{x}) \in$ $L^{2}\left(\boldsymbol{T}_{i}, \mathrm{~d} \mu^{\varepsilon}\right)$ yields $u^{(1)}(\boldsymbol{x}) \zeta_{i}(\boldsymbol{x}) \in H_{0}^{1}\left(\boldsymbol{T}_{i}\right)$. Hence,

$$
u^{(1)}(\boldsymbol{x})=\sum_{i=1}^{m} u^{(1)}(\boldsymbol{x}) \zeta_{i}(\boldsymbol{x}) \in H^{1}(\mathcal{T}) \equiv H_{\mathrm{per}}^{1}(\boldsymbol{T})
$$

\section{Appendix C. Proof of the equality (2.20)}

In our proof of the equality (2.20) we follow the argument of Zhikov [28, lemmas 6.1 and 4.3$]$.

Let us consider test functions of the form

$$
\psi(\boldsymbol{x})=\psi^{\varepsilon}(\boldsymbol{x})=\varepsilon \phi(\boldsymbol{x}) h\left(\varepsilon^{-1} \tilde{\boldsymbol{x}}\right),
$$

where $\phi(\boldsymbol{x}) \in C_{\text {per }}^{\infty}(\boldsymbol{T})$ and $h \in C_{\text {per }}^{\infty}\left(Q_{2}\right)$. Then

$$
\nabla \psi^{\varepsilon}(\boldsymbol{x})=\left.\phi(\boldsymbol{x}) \nabla_{y} h(\tilde{\boldsymbol{y}})\right|_{\tilde{y}=\varepsilon^{-1} \tilde{x}}+\varepsilon h\left(\varepsilon^{-1} \tilde{\boldsymbol{x}}\right) \nabla \phi(\boldsymbol{x}) .
$$


We substitute $\psi^{\varepsilon}(\boldsymbol{x})$ into the original integral identity (2.3) and obtain the following equality:

$$
\begin{aligned}
\int_{\boldsymbol{T} \cap F_{1}^{\varepsilon}} \phi(\boldsymbol{x}) & \left.\nabla u^{\varepsilon}(\boldsymbol{x}) \cdot \nabla_{y} h(\tilde{\boldsymbol{y}})\right|_{\tilde{y}=\varepsilon^{-1} \tilde{x}} \mathrm{~d} \boldsymbol{x} \\
& +\varepsilon \int_{\boldsymbol{T} \cap F_{1}^{\varepsilon}} h\left(\varepsilon^{-1} \tilde{\boldsymbol{x}}\right) \nabla u^{\varepsilon}(\boldsymbol{x}) \cdot \nabla \phi(\boldsymbol{x}) \mathrm{d} \boldsymbol{x} \\
& +\varepsilon^{2} \int_{\boldsymbol{T} \cap F_{0}^{\varepsilon}}\left[u_{, 1}^{\varepsilon}(\boldsymbol{x})\left(\left.\phi(\boldsymbol{x}) h_{, 1}(\tilde{\boldsymbol{y}})\right|_{\tilde{y}=\varepsilon^{-1} \tilde{x}}+\varepsilon h\left(\varepsilon^{-1} \tilde{\boldsymbol{x}}\right) \phi_{, 1}(\boldsymbol{x})\right)\right. \\
& \left.+u_{, 2}^{\varepsilon}(\boldsymbol{x})\left(\left.\phi(\boldsymbol{x}) h_{, 2}(\tilde{\boldsymbol{y}})\right|_{\tilde{y}=\varepsilon^{-1} \tilde{x}}+\varepsilon h\left(\varepsilon^{-1} \tilde{\boldsymbol{x}}\right) \phi_{, 2}(\boldsymbol{x})\right)\right] \mathrm{d} \boldsymbol{x} \\
& +\varepsilon \int_{\boldsymbol{T} \cap F_{0}^{\varepsilon}} u_{, 3}^{\varepsilon}(\boldsymbol{x}) h\left(\varepsilon^{-1} \tilde{\boldsymbol{x}}\right) \phi_{, 3}(\boldsymbol{x}) \mathrm{d} \boldsymbol{x}+\lambda \varepsilon \int_{\boldsymbol{T}} u^{\varepsilon}(\boldsymbol{x}) \phi(\boldsymbol{x}) h\left(\varepsilon^{-1} \tilde{\boldsymbol{x}}\right) \mathrm{d} \boldsymbol{x} \\
= & \varepsilon \int_{\boldsymbol{T}} f(\boldsymbol{x}) \phi(\boldsymbol{x}) h\left(\varepsilon^{-1} \tilde{\boldsymbol{x}}\right) \mathrm{d} \boldsymbol{x} .
\end{aligned}
$$

Using the a priori estimates obtained in $\S 2.2$, it is easy to see that, in the last identity, all terms but the first vanish as $\varepsilon \rightarrow 0$. Hence, the first term also vanishes, i.e.

$$
\left.\int_{\boldsymbol{T} \cap F_{1}^{\varepsilon}} \phi(\boldsymbol{x}) \nabla u^{\varepsilon}(\boldsymbol{x}) \cdot \nabla_{y} h(\tilde{\boldsymbol{y}})\right|_{\tilde{y}=\varepsilon^{-1} \tilde{x}} \mathrm{~d} \boldsymbol{x} \stackrel{\varepsilon \rightarrow 0}{\longrightarrow} 0 .
$$

Denote $\mathrm{d} \mu_{1}^{\varepsilon}:=\left.\mathrm{d} \boldsymbol{x}\right|_{F_{1}^{\varepsilon}}$. It is proved by Zhikov [28, theorem 4.2] that, due to boundedness of the sequence $\nabla u^{\varepsilon}$ in the space $L^{2}\left(\boldsymbol{T}, \mathrm{d} \mu_{1}^{\varepsilon}\right)$, the following convergence holds:

$$
\nabla u^{\varepsilon}(\boldsymbol{x}) \stackrel{2}{\rightarrow} \nabla u^{(1)}(\boldsymbol{x})+\boldsymbol{r}(\boldsymbol{x}, \boldsymbol{y}) \text { with respect to } \mathrm{d} \mu_{1},
$$

where $\boldsymbol{r}(\boldsymbol{x}, \boldsymbol{y}) \in L^{2}\left(\boldsymbol{T}, V_{\text {pot }}\left(Q, \mathrm{~d} \mu_{1}\right)\right) .{ }^{14}$ In particular,

$$
\nabla u^{\varepsilon}(\boldsymbol{x}) \rightarrow \int_{Q}\left(\nabla u^{(1)}(\boldsymbol{x})+\boldsymbol{r}(\boldsymbol{x}, \boldsymbol{y})\right) \mathrm{d} \mu_{1} \quad \text { in }\left[L^{2}(\boldsymbol{T})\right]^{3} .
$$

Due to (C2),

$$
\begin{aligned}
&\left.\int_{\boldsymbol{T}} \phi(\boldsymbol{x}) \nabla u^{\varepsilon}(\boldsymbol{x}) \cdot \nabla_{y} h(\tilde{\boldsymbol{y}})\right|_{y=\varepsilon^{-1} x} \mathrm{~d} \mu_{1}^{\varepsilon} \\
& \stackrel{\varepsilon \rightarrow 0}{\longrightarrow} \int_{\boldsymbol{T}} \int_{Q}\left(\nabla u^{(1)}(\boldsymbol{x})+\boldsymbol{r}(\boldsymbol{x}, \boldsymbol{y})\right) \cdot \nabla_{y} h(\tilde{\boldsymbol{y}}) \phi(\boldsymbol{x}) \mathrm{d} \mu_{1} \mathrm{~d} \boldsymbol{x}
\end{aligned}
$$

and, in view of $(\mathrm{C} 1)$,

$$
\int_{\boldsymbol{T}} \int_{Q}\left(\nabla u^{(1)}(\boldsymbol{x})+\boldsymbol{r}(\boldsymbol{x}, \boldsymbol{y})\right) \cdot \nabla_{y} h(\tilde{\boldsymbol{y}}) \phi(\boldsymbol{x}) \mathrm{d} \mu_{1} \mathrm{~d} \boldsymbol{x}=0
$$

for arbitrary $\phi(\boldsymbol{x}) \in C_{\text {per }}^{\infty}(\boldsymbol{T})$ and $h \in C_{\text {per }}^{\infty}\left(Q_{2}\right)$. Thus,

$$
\int_{Q}\left(\nabla u^{(1)}(\boldsymbol{x})+\boldsymbol{r}(\boldsymbol{x}, \boldsymbol{y})\right) \cdot \nabla_{y} h(\tilde{\boldsymbol{y}}) \mathrm{d} \mu_{1}=0
$$

${ }^{14} \mathrm{By}$ definition, the set $V_{\mathrm{pot}}\left(Q, \mathrm{~d} \mu_{1}\right)$ of periodic potential vectors is the closure of the set $\left\{\nabla \phi: \phi \in C_{\text {per }}^{\infty}(Q)\right\}$ in the space $\left[L_{\text {per }}^{2}\left(Q, \mathrm{~d} \mu_{1}\right)\right]^{3}$. 
for any $h \in C_{\text {per }}^{\infty}\left(Q_{2}\right)$ or, rewriting it in a more convenient form,

$$
\int_{Q_{2}}\left(\nabla_{\tilde{x}} u^{(1)}(\boldsymbol{x})+\langle\tilde{\boldsymbol{r}}(\boldsymbol{x}, \boldsymbol{y})\rangle_{y_{3}}\right) \cdot \nabla_{\tilde{y}} h(\tilde{\boldsymbol{y}}) \mathrm{d} \tilde{\mu}_{1}=0
$$

where $\tilde{\mu}_{1}:=\left.\mathrm{d} \tilde{\boldsymbol{y}}\right|_{\tilde{F}_{1} \cap Q_{2}}$. Taking into account (C 3), we obtain

$$
\nabla u^{\varepsilon}(\boldsymbol{x}) \rightarrow\left(\int_{Q_{2}}\left(\nabla_{\tilde{x}} u^{(1)}(\boldsymbol{x})+\langle\tilde{\boldsymbol{r}}(\boldsymbol{x}, \boldsymbol{y})\rangle_{y_{3}}\right) \mathrm{d} \tilde{\mu}_{1}, \mathfrak{f}_{1} u_{, 3}^{(1)}(\boldsymbol{x})+\int_{Q_{2}}\left\langle r_{3}(\boldsymbol{x}, \boldsymbol{y})\right\rangle_{y_{3}} \mathrm{~d} \tilde{\mu}_{1}\right) .
$$

But $\left\langle r_{3}(\boldsymbol{x}, \boldsymbol{y})\right\rangle_{y_{3}}=0$, due to the fact that $\boldsymbol{r}(\boldsymbol{x}, \boldsymbol{y}) \in L^{2}\left(\boldsymbol{T}, V_{\text {pot }}\left(Q, \mathrm{~d} \mu_{1}\right)\right)$ and therefore the spaces $V_{\text {pot }}\left(Q, \mathrm{~d} \mu_{1}\right)$ and $V_{\text {pot }}\left(Q, \mathrm{~d} \tilde{\mu}_{1} \times \mathrm{d} y_{3}\right)$ coincide. Hence,

$$
\begin{aligned}
\nabla u^{\varepsilon}(\boldsymbol{x})-\left(\int_{Q_{2}}\left(\nabla_{\tilde{x}} u^{(1)}(\boldsymbol{x})+\langle\tilde{\boldsymbol{r}}(\boldsymbol{x}, \boldsymbol{y})\rangle_{y_{3}}\right)\right. & \left.\mathrm{d} \tilde{\mu}_{1}, \mathfrak{f}_{1} u_{, 3}^{(1)}(\boldsymbol{x})\right) \\
= & \left(A_{2 \mathrm{D}}^{\mathrm{hom}} \nabla_{\tilde{x}} u^{(1)}(\boldsymbol{x}), \mathfrak{f}_{1} u_{, 3}^{(1)}(\boldsymbol{x})\right),
\end{aligned}
$$

where the matrix $A_{2 \mathrm{D}}^{\text {hom }}$ is given by the formula (2.7). The last equality in (C 5) is due to (C4).

\section{References}

1 G. Allaire. Homogenization and two-scale convergence. SIAM J. Math. Analysis 23 (1992), $1482-1518$.

2 G. Allaire and F. Murat. Homogenization of the Neumann problem with nonisolated holes. Asymp. Analysis $\mathbf{7}$ (1993), 81-95.

3 Y. Amirat, K. Hamdache and A. Ziani. Homogénéisation non locale pour des équations dégénérées à coefficients périodiques. C. R. Acad. Sci. Paris I 312 (1991), 963-966.

4 N. S. Bakhvalov and G. P. Panasenko. Homogenization: averaging processes in periodic media (Moscow: Nauka, 1984). (In Russian.) (Engl. transl. Mathematics and its applications (Soviet Series) vol. 36 (Dordrecht: Kluwer Academic, 1989).

5 A. Bensoussan, J.-L. Lions and G. C. Papanicolaou. Asymptotic analysis for periodic structures. (Amsterdam: North Holland, 1978).

6 G. Bouchitté and M. Bellieud. Homogenization of a soft elastic material reinforced by fibers. Asymp. Analysis 32 (2002), 153-183.

7 M. Briane. Homogenization of non-uniformly bounded operators: critical barrier for nonlocal effects. Arch. Ration. Mech. Analysis 164 (2002), 73-101.

8 M. Camar-Eddine and G. W. Milton. Non-local interactions in the homogenization closure of thermoelectric functionals. Asymp. Analysis 41 (2004), 259-276.

9 K. D. Cherednichenko. Higher-order and non-local effects in homogenisation of periodic media. PhD thesis, University of Bath (2001). (Available at http://www.maths.ox.ac.uk/ cheredni/.)

10 K. D. Cherednichenko. Two-scale asymptotics for nonlocal effects in composites with highly anisotropic fibres. Preprint (2005). (Available at http://www.damtp.cam.ac.uk/ user $/ \mathrm{kdc} 20 /$.)

11 K. D. Cherednichenko and V. P. Smyshlyaev. On full two-scale expansion of the solutions of nonlinear periodic rapidly oscillating problems and higher-order homogenised variational problems. Arch. Ration. Mech. Analysis 174 (2004), 385-442.

12 V. N. Fenchenko and E. Ya. Khruslov. Asymptotic behaviour or the solutions of differential equations with strongly oscillating and degenerating coefficient matrix. Dokl. Akad. Nauk Ukrain SSSR A 4 (1980), 26-30. (In Russian.)

13 N. A. Fleck, G. M. Muller, M. F. Ashby and J. W. Hutchinson. Strain gradient plasticity: theory and experiment. Acta Metall. Mater. 42 (1994), 475-487.

14 I. S. Gradshteyn and I. M. Ryzhik. Table of integrals, series, and products (Academic, 2000). 
15 V. V. Jikov, S. M. Kozlov and O. A. Oleinik. Homogenization of differential operators and integral functionals (Springer, 1994).

16 E. Ya. Khruslov. An averaged model of a strongly inhomogeneous medium with memory. Usp. Mat. Nauk 45 (1990), 197-198. (Engl. transl. Russ. Math. Surv. 45 (1990), 211-212.)

P. Kuchment. The mathematics oh photonic crystals. In Mathematical models in optical science, Frontiers in Applied Mathematics, vol. 22, ch. 7, pp. 207-272 (Philadelphia, PA: SIAM, 2001).

18 G. Nguetseng. A general convergence result for a functional related to the theory of homogenization. SIAM J. Math. Analysis 20 (1989), 608-623.

19 G. P. Panasenko. Multicomponent homogenization of processes in strongly nonhomogeneous structures. Mat. USSR Sb. 69 (1991), 143-153.

20 A. Sili. Homogenization of a nonlinear monotone problem in an anisotropic medium. Math. Models Meth. Appl. Sci. 14 (2004), 329-353.

21 V. P. Smyshlyaev and K. D. Cherednichenko. On derivation of strain gradient effects in the overall behaviour of periodic heterogeneous media. J. Mech. Phys. Solids 48 (2000), $1325-1357$.

22 P. St J. Russell. Photonic crystal fibers. Science 299 (2003), 358-362.

23 L. Tartar. Nonlocal effects induced by homogenisation. In Partial differential equations and the calculus of variations, II. Progress in Nonlinear Differential Equations and their Applications, vol. 2, pp. 925-938. (Boston, MA: Birkhäuser, 1989).

24 L. Tartar. Memory effects and homogenisation. Arch. Ration. Mech. Analysis 111 (1990), $121-133$

25 J. Wermer. Potential theory. Lecture Notes in Mathematics, vol. 408 (Springer, 1981).

26 J. R. Willis. The overall elastic response of composite materials. J. Appl. Mech. 50 (1983), $1202-1209$.

27 K. Yosida. Functional analysis (Springer, 1971).

28 V. V. Zhikov. On an extension of the method of two-scale convergence and its applications. Sb. Math. 191 (2000), 973-1014.

(Issued 17 February 2006) 